\title{
Simulation-Optimization Approach for the Production and Distribution Planning Problem in the Green Closed-Loop Supply Chain
}

Niloofar Nadim Kabiri

Babol Noshirvani University of Technology

Saeed Emami ( $\nabla$ s_emami@nit.ac.ir)

Babol Noshirvani University of Technology https://orcid.org/0000-0002-6356-0529

Abdul Sattar Safaei

Babol Noshirvani University of Technology

\section{Research Article}

Keywords: Production and Distribution Planning, Simulation- Optimization, Green Closed-Loop Supply Chain, Monte Carlo Simulation, Three-level Bi-objective Programming, NSGA-II Algorithm

Posted Date: October 21st, 2021

DOI: https://doi.org/10.21203/rs.3.rs-836044/v1

License: (c) (i) This work is licensed under a Creative Commons Attribution 4.0 International License. Read Full License 


\title{
Simulation-optimization approach for the production and distribution planning problem in the green closed-loop supply chain
}

\author{
Niloofar Nadim Kabiri, Saeed Emami ${ }^{*}$, Abdul Sattar Safaei \\ Department of Industrial Engineering, Babol Noshiravani University of Technology, Babol, \\ 47148-71167, IRAN \\ niloo.nadim@stu.nit.ac.ir, s_emami@nit.ac.ir,s.safaei@nit.ac.ir
}

\begin{abstract}
With the growth of multinational companies, increasing international and domestic competition between companies, upgrading information technology, and increasing customer expectations, accurate supply chain (SC) planning is essential. In such an environment, pollution has become more severe in recent decades, and with the weakening of the environment and global warming, green SC management (GSCM) strategies have become more attention in recent decades. In this research, we consider the integrated production and distribution (PD) planning problem of a multi-level green closed-loop SC (GCLSC) system, which includes multiple recycling, manufacturing/ remanufacturing, and distribution centers. We present a three-level bi-objective programming model to maximize profit and minimize the amount of greenhouse gas emissions. A hierarchical iterative approach utilizing the LP-metric method and the non-dominated sorting genetic algorithm (NSGA-II) is introduced to solve the proposed model. Also, the Taguchi approach is applied to find optimum control parameters of NSGA-II. Moreover, Monte Carlo (MC) simulation is applied to tackle uncertainty in demand, and the NSGA-II algorithm is fusioned with MC simulation (MCNSGA-II). The results obtained show that the simulationoptimization approach presented better results than the deterministic approach.
\end{abstract}

Keywords: Production and Distribution Planning, Simulation- Optimization, Green ClosedLoop Supply Chain, Monte Carlo Simulation, Three-level Bi-objective Programming, NSGA-II Algorithm

\section{1- Introduction}

Many companies strive to meet their expectations and customer satisfaction to modify and expand their business processes so that they can successfully challenge the growth of competitive environments. Therefore, SC management (SCM) is an attractive topic (Ramezani et al. 2014). An SC contains all activities that convert raw materials into final products and transfer 
them to customers. Production planning and distribution planning are the two core optimization problems in SCM, which are always linked together (Jing and Li 2018), (Kang et al. 2016). Production planning includes decisions regarding usual time and overtime production, hiring and firing of labor, machine capacity, and subcontracting are made for a planning horizon. Distribution planning decisions are related to determining which facilities would provide to the demands of which markets (Fahimnia et al. 2013).

In the past, the elements of an SC, such as manufacturers and distributors, have been considered as discrete commerce entities looking for their maximum profits (Barbarosoğlu and Özgür 1999). Nevertheless, this may lead to problems between companies in a SC. Because the benefits of the firms cannot be balanced and maximizing profits for individual companies does not mean maximizing profits for the entire SC. Therefore, the overall efficiency and operational performance of the SC depend on integrated PD planning decisions (Liang and Cheng 2009).

In the past few years, topics like green remanufacturing and manufacturing, waste management, and reverse logistics as important subsets of GSCM have received a lot of attention. Note that the integration of environmental consideration into SCM specifies GSCM (Srivastava 2008).

Optimization of fuel consumption, gas emission reduction, and waste management are important in the GCLSC network design problem (Lin et al. 2014). Today, gas emissions is an important environmental issue; thus, transportation has been considered by green logistics researchers (Dekker et al. 2012).

Moreover, product recycling has faced increasing attention throughout the years. Today, more companies employ voluntarily and positively in the products recycling and remanufacturing business by considering economic benefits. Accordingly, the common forward $\mathrm{SC}$ is converted into the closed-form through integrating recycling and remanufacturing (Jing and $\mathrm{Li} 2018$ ).

Consequently, in this study, we investigate the integrated PD planning problem for the GCLSC, which is multi-product, multi-period, and multi-level. There are many decision-makers at different levels of the GCLSC that have various decision-making authority and objectives. The set of strategies and the goal attainment of lower levels can be affected by the decisions from the upper levels. Nevertheless, lower levels have considerable autonomy, so the upper levels cannot totally control them. Generally, each level of the SC is finding the best decision considering the decisions of the other levels. Therefore, multi-level modeling of the problem is considered with the aim to maximize profit and minimize adverse environmental impacts at each level. We present a multi-level bi-objective programming model that includes multiple recycling, manufacturing/remanufacturing factories, and distribution centers. A hierarchical iterative approach using the LP-metric method for small-size instances and the NSGA-II algorithm for large-size instances is suggested to solve the proposed model. Moreover, the MCNSGA-II algorithm is developed to tackle uncertainty in demand. The results obtained illustrate that the MCNSGA-II algorithm introduced better results than the NSGA-II algorithm.

The paper is structured as follows: In Section 2, the related literature is reviewed. The considered problem, its assumption, and the suggested multi-level bi-objective model are defined in Section 3 in detail. The solution approach and the computational results are presented in Section 4 and Section 5, respectively. Section 6 contains conclusions and some suggestions for future works.

\section{2- Literature review}


The analysis and design of PD systems have been a vital area of research over the years. Geoffrion and Graves (1974) studied a single-period PD problem and suggested a solution approach based on the Benders decomposition algorithm. This is probably the first article to suggest a general mixed-integer programming (MIP) model for the mentioned problem. Haq et al. (1991) utilized an integrated production-inventory-distribution planning formulation in a large fertilizer industry in North India that incorporates many realistic conditions such as lead times, set-up cost, and recycling of production losses as well as backlogging. Chen and Wang (1997) suggested an integrated framework for steel PD planning at a major steel producer company in Canada. Ozdamar and Yazgac (1999) introduced a hierarchical PD planning method for a multinational plant with multiple warehouses. Lee and Kim (2000) presented a general PD model in the literature and offered a solution method using a hybrid approach combining analytical and simulation techniques. Varthanan et al. (2012) investigated PD problem with stochastic demand to minimize regular, overtime and outsourced production costs. They used a simulation based heuristic discrete particle swarm optimization (DPSO) algorithm to solve the problem.

Nasiri et al. (2014) suggested a PD model for a three-level program with uncertain demands. They presented a solution framework based on the Lagrangian Relaxation algorithm, which is developed by a heuristic to solve sub-problems. Niknamfar et al. (2015) considered a three-level SC with multiple production and distribution centers, and different customer areas. They presented a robust counterpart model in PD planning to minimize the total cost. Sarrafha et al. (2015) suggested a bi-objective mixed-integer non-linear programming (MINLP) formulation to design an SC network that includes suppliers, production and distribution centers, and retailers. They used the multi-objective biogeography based optimization (MOBBO) algorithm for solving the problem. Seyedhosseini and Ghoreyshi (2015) introduced a mathematical formulation for integrated PD planning for perishable products. An innovative framework is used to solve the formulation in which the suggested method first solves the production problem and afterward, the distribution problem. Devapriya et al. (2017) considered a PD planning problem of a perishable product and provided a MIP model to minimize cost. They presented a solution approach using evolutionary algorithms to solve the model.

Zamarripa et al. (2016) introduced a rolling horizon approach for coordinating the PD of industrial gas SC. Zheng et al. (2016) presented a penalty function-based method to solve a riskaverse PD planning problem. The method changes the formulation into some optimization problems which can be solved by traditional optimization software. Ma et al. (2016) proposed a PD planning model applying bi-level programming for SCM and developed a genetic algorithm to solve the model. Rezaeian et al. (2016) suggested an MINLP model for the integrated PD and inventory planning for perishable products with a fixed lifetime all over a two-echelon SC by integrating production centers and distributors. Moon et al. (2016) introduced a bi-objective MIP model to design a four-stage distribution system under a carbon emission constraint. They proposed a two-phase method to solve the model and find a non-dominated solution.

Osorio et al. (2017) suggested a simulation-optimization formulation to make strategic and operational decisions in production planning. They used discrete event simulation to demonstrate the flows across the SC, and MIP model to assist daily decisions. Wei et al. ( 2017) studied the 
integrated PD planning problem and presented a model with a two-stage production structure. They applied relax-and-fix and fix-and-optimize approaches to solve the problem. Ensafian and Yaghoubi (2017) considered an SC that consists of procurement, production, and distribution of platelets. They presented a bi-objective mathematical model to maximize the freshness of the platelets and minimize the total cost. Moreover, a robust optimization approach is used to tackle uncertain demand. Farahani and Rahmani (2017) proposed a MIP model that includes production planning, allocation-location facilitation, and distribution planning to maximize the profit of a crude oil network. Nourifar et al. (2018) introduced a multi-period decentralized SC network model with uncertainty. Uncertainty parameters such as demand and final product prices were defined by stochastic and fuzzy numbers. They presented a solution framework based on the Kth-best algorithm, chance constraint approach, and fuzzy approach.

Rafiei et al. (2018) investigated a PD planning problem within a four-echelon SC. The problem is modeled in two non-competitive and competitive markets to minimize total chain cost and maximize service level. Casas-Ramírez et al. (2018) studied an SC, including factories and depots. They proposed a MIP model to balance the total workload and minimize the total cost of the SC. To solve this model, they used an adapted bi-objective GRASP to find non-dominated solutions. Jing and $\mathrm{Li}$ (2018) considered a multi-echelon closed-loop SC planning problem involving a joint recycling center, multiple remanufacturing/manufacturing centers, and multiple distribution centers decentralized to various areas. The solution framework was designed by a hierarchical iterative approach based on the self-adaptive genetic algorithm. Goodarzian et al. (2021) studied a novel multi-objective formulation is devised for the PD problem of a supply chain that consists of several suppliers, manufacturers, distributors, and different customers. Due to the NP-hardness of problems, NSGA-II and Fast PGA algorithm are applied. Pant et al. (2021) developed a bi-objective CLSC model for the paper industry under uncertain environment. The first objective of the proposed model is to maximize SC surplus, and the second objective is to incorporate sustainability through minimizing carbon content by reducing the number of trucks between various echelons of CLSC network. They considered uncertainty at demand points and applied MC simulation to handle it.

The reviewed articles on integrated production-distribution planning are summarized in Table 1 . The last row of Table 1 is for the present study. Specifically, the followings are the significant contributions of this paper:

- A three-level bi-objective programming model is presented to maximize profit and minimize the amount of greenhouse gas emissions.

- NSGA-II algorithm is developed to solve the bi-objective model at each level.

- A hierarchical iterative approach is applied to solved the three-level model.

- NSGA-II algorithm is combined with MC simulation (MCNSGA-II) to tackle uncertainty in demand. 
Table 1: The representative works on the integrated production-distribution planning

\begin{tabular}{|c|c|c|c|c|c|c|c|c|c|c|c|c|}
\hline \multirow{2}{*}{ Authors } & \multirow{2}{*}{ Year } & \multicolumn{2}{|c|}{ Product } & \multicolumn{2}{|c|}{ Period } & \multicolumn{2}{|c|}{ Objective } & \multicolumn{2}{|c|}{ Parameter } & \multirow{2}{*}{$\begin{array}{l}\text { Uncertain } \\
\text { solution } \\
\text { method }\end{array}$} & \multirow{2}{*}{ Solution Method } & \multirow{2}{*}{ Software } \\
\hline & & Single & Multi & Single & Multi & Single & Multi & Certain & Uncertain & & & \\
\hline Geoffrion and Graves (1974) & 1974 & & $*$ & $*$ & & $*$ & & $*$ & & - & Bender method & - \\
\hline Haq et al. (1991) & 1991 & & $*$ & & $*$ & $*$ & & $*$ & & - & Exact & - \\
\hline Chen and Wang (1997) & 1997 & & $*$ & $*$ & & $*$ & & $*$ & & - & Exact & - \\
\hline Ozdamar and Yazgac (1999) & 1999 & & $*$ & & $*$ & $*$ & & $*$ & & - & Exact & GAMS \\
\hline Lee and Kim (2000) & 2000 & & $*$ & & $*$ & $*$ & & & $*$ & Simulation & Exact & GAMS \\
\hline Varthanan et al. (2012) & 2012 & & $*$ & & $*$ & $*$ & & & $*$ & Simulation & DPSO & MATLAB \\
\hline Nasiri et al. (2014) & 2014 & & $*$ & & $*$ & $*$ & & & $*$ & Stochastic & GA & MATLAB \\
\hline Niknamfar et al. (2015) & 2014 & & $*$ & & $*$ & $*$ & & & $*$ & Robust- fuzzy & Exact & GAMS \\
\hline Sarrafha et al. (2015) & 2015 & $*$ & & & $*$ & & $*$ & $*$ & & - & MOPSO, NSGA-II & MATLAB \\
\hline $\begin{array}{l}\text { Seyedhosseini and Ghoreyshi } \\
\text { (2015) }\end{array}$ & 2015 & $*$ & & & * & * & & $*$ & & - & Heuristic algorithm & MATLAB \\
\hline Devapriya et al. (2017) & 2016 & $*$ & & & $*$ & $*$ & & $*$ & & - & GA, MA & MATLAB \\
\hline Zamarripa et al. (2016) & 2016 & & * & & $*$ & $*$ & & $*$ & & - & $\begin{array}{l}\text { Rolling Horizon } \\
\text { Algorithm }\end{array}$ & GAMS \\
\hline Zheng et al. (2016) & 2016 & $*$ & & $*$ & & $*$ & & $*$ & & - & Exact & GAMS \\
\hline Ma et al. (2016) & 2016 & & $*$ & $*$ & & $*$ & & & $*$ & Fuzzy & GA & MATLAB \\
\hline Rezaeian et al. (2016) & 2016 & & $*$ & & $*$ & $*$ & & $*$ & & - & GA, SA & MATLAB \\
\hline Moon et al. (2016) & 2016 & $*$ & & & $*$ & & $*$ & $*$ & & - & Exact & CPLEX \\
\hline Osorio et al. (2017) & 2016 & & $*$ & & $*$ & $*$ & & & $*$ & Simulation & Exact & JAVA \\
\hline Wei et al. ( 2017) & 2017 & & $*$ & & $*$ & $*$ & & $*$ & & - & Exact & CPLEX \\
\hline $\begin{array}{c}\text { Ensafian and Yaghoubi } \\
\text { (2017) }\end{array}$ & 2017 & $*$ & & & $*$ & & $*$ & & $*$ & Robust & Exact & GAMS \\
\hline Farahani and Rahmani (2017) & 2017 & & $*$ & & $*$ & $*$ & & $*$ & & - & Exact & GAMS \\
\hline Nourifar et al. (2018) & 2017 & & $*$ & & $*$ & $*$ & & & $*$ & $\begin{array}{l}\text { Stochastic - } \\
\text { fuzzy }\end{array}$ & Exact & LINGO \\
\hline Rafiei et al. (2018) & 2018 & $*$ & & & $*$ & & $*$ & $*$ & & - & Exact & LINGO \\
\hline Casas-Ramírez et al. (2018) & 2018 & $*$ & & $*$ & & & $*$ & $*$ & & - & GRASP & $\mathrm{C}++$ \\
\hline Jing and Li (2018) & 2018 & & $*$ & & $*$ & $*$ & & $*$ & & - & SAGA & MATLAB \\
\hline Goodarzian et al. (2021) & 2021 & & $*$ & & $*$ & & $*$ & $*$ & & - & $\begin{array}{l}\text { NSGA-II and Fast } \\
\text { PGA }\end{array}$ & MATLAB \\
\hline Pant et al. (2021) & 2021 & & $*$ & & $*$ & & $*$ & & $*$ & $\begin{array}{l}\text { Monte Carlo } \\
\text { Simulation }\end{array}$ & $\begin{array}{l}\text { Augmented epsilon } \\
\text { method }\end{array}$ & MATLAB \\
\hline Present study & 2021 & & $*$ & & $*$ & & $*$ & & $*$ & $\begin{array}{l}\text { Monte Carlo } \\
\text { Simulation }\end{array}$ & $\begin{array}{c}\text { LP-metric } \\
\text { NSGA-II and } \\
\text { MCNSGA-II } \\
\end{array}$ & $\begin{array}{l}\text { GAMS and } \\
\text { MATLAB }\end{array}$ \\
\hline
\end{tabular}




\section{3- Mathematical modeling}

In this paper, a three-level bi-objective programming model is presented for a GCLSC, which includes multiple recycling centers, multiple manufacturing/remanufacturing factories, and multiple distributors.

Multi-level programming is an optimization approach that has a multi-layer hierarchical form. In this form, decision-makers of different levels have various decision-making authority and objectives. The set of strategies and the goal attainment of lower levels can be affected by the decisions from the upper levels. Nevertheless, lower levels have considerable autonomy, so the upper levels cannot totally control them (Jing and Li 2018). Regarding the basic concept of multi-level programming, each subordinate level in this model is situated in different region and has its policies and strategies. The first-level decision maker (recycling centers) sets own goals and/or decision, and then asks each subordinate level of the organization for their optima, that is calculated in isolation. Because of it, Multi-level programming is used.

In the considered GCLSC, returned products are disassembled to components in the multiple recycling centers. Testing activities to know the defective components perform in the centers. The components can be saved in the inventory of eligible components if they have a remanufacturing standard; otherwise they will be excreted. Eligible components are carried to various remanufacturing/ manufacturing centers.

The remanufacturing/manufacturing centers test the quality and efficiency of Eligible components, or raw materials are converted to new components. Remanufactured and new components are used to produce remanufactured and new products, respectively. The products are added to the inventory of remanufactured and new products and are carried to various distribution centers according to orders.

Each distribution center can select one or more remanufacturing/manufacturing centers for satisfying demand, and provide remanufactured or new products to retailers for sales. Moreover, the returned products from retailers are gathered and recycled by distribution centers and carried to multiple recycling centers. Note that there are several kinds of vehicles to transport parts and products between the GCLSC components.

Furthermore, the assumptions for the considered problem are as follows:

1. The SC is multi-product, multi-period, and multi-level.

2. Demand for remanufactured and new products is uncertain and can only be met by themselves, and remanufactured products have lower selling prices than new products.

3. The production cost of each factory is different.

4. Vehicles have variable capacity in each level.

5. The cost of transfer between the nodes is different.

6. Processing and reprocessing parts in the manufacturing/remanufacturing factories lead to greenhouse gas emissions into the environment.

7. The transportation of vehicles at each level in each period has a greenhouse gas emissions limit.

Figure 1 shows a schematic of the considered SC. 


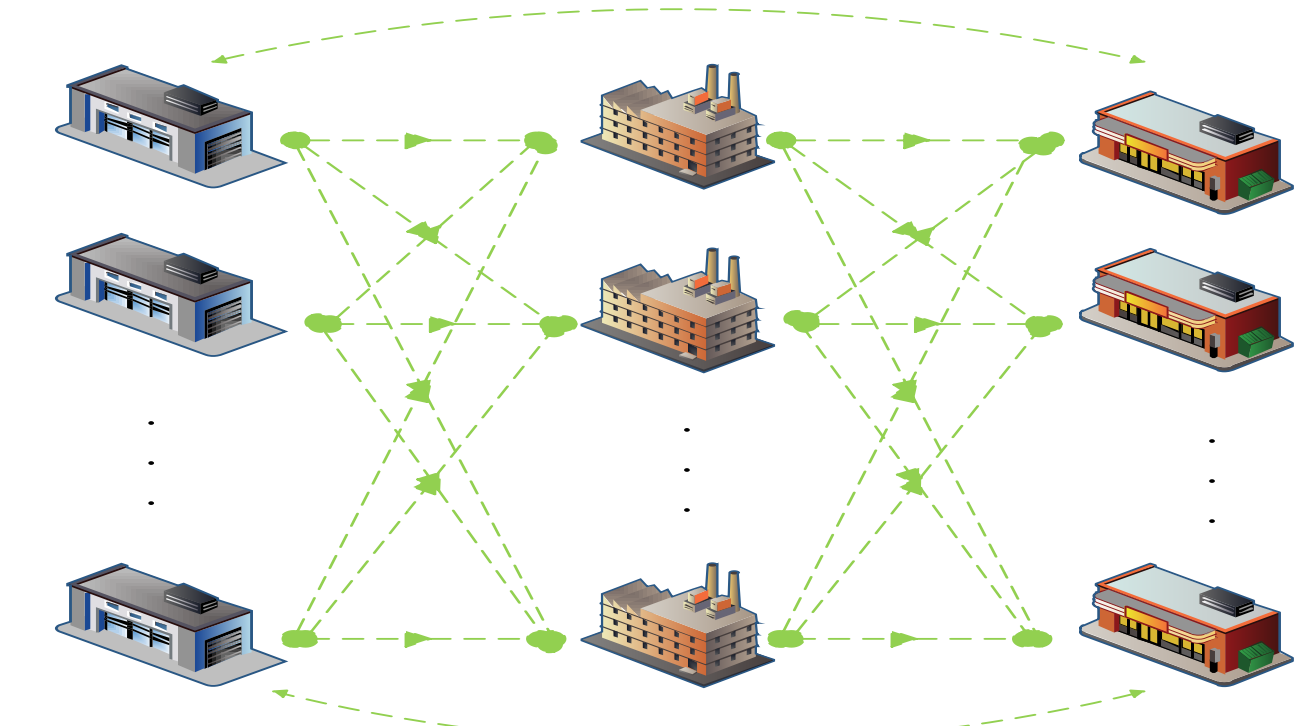

First level: the recycling
centers $\begin{gathered}\text { Second level: the manufacturing/ Third level: distributors } \\ \text { remanufacturing factories }\end{gathered}$

Figure 1: The schematic of the considered GCLSC

The notations are introduced in Table 13 to Table 19 in the Appendix. The model of each level is as below.

\section{3-1- The recycling centers, the first level}

The bi-objective MIP model at the first level (FLM) to maximize profit and minimize adverse environmental effects is as follows:

$$
\begin{aligned}
& \max F_{1}^{R}=\sum_{k=1}^{K} \sum_{i=1}^{I} \sum_{c=1}^{C} \sum_{v=1}^{V} \sum_{t=1}^{T} P P C_{k i c t} \cdot a f_{k i c v t}-\left\{\sum_{j=1}^{J} \sum_{k=1}^{k} \sum_{p=1}^{P} \sum_{v=1}^{V} \sum_{t=1}^{T} U R C C_{j k p t} \cdot d a_{j k p v t}+\right. \\
& \sum_{k=1}^{K} \sum_{p=1}^{P} \sum_{t=1}^{T} S D T_{k p t} \cdot \sigma_{k p t}+\sum_{k=1}^{K} \sum_{p=1}^{P} \sum_{t=1}^{T} U D T C_{k p t} \cdot d t_{k p t}+\sum_{k=1}^{K} \sum_{c=1}^{C} \sum_{t=1}^{T} U D C_{k c t} \cdot d_{k c t}+ \\
& \left.\sum_{k=1}^{K} \sum_{p=1}^{P} \sum_{t=1}^{T} I C R P_{k p t}^{R} \cdot \alpha_{k p t}^{R}+\sum_{k=1}^{K} \sum_{c=1}^{C} \sum_{t=1}^{T} I C Q C_{k c t}^{R} \cdot \beta_{k c t}^{R}+\sum_{k=1}^{K} \sum_{i=1}^{I} \sum_{c=1}^{C} \sum_{v=1}^{V} \sum_{t=1}^{T} U T C_{k i c v t}^{R F} \cdot a f_{k i c v t}\right\} \\
& \min F_{2}^{R}=\sum_{k=1}^{K} \sum_{i=1}^{I} \sum_{c=1}^{C} \sum_{v=1}^{V} \sum_{t=1}^{T} d i s_{k i}^{R F} \cdot \text { Emis }_{k i c v t}^{R F} \cdot a f_{k i c v t}
\end{aligned}
$$

s.t.

$$
\begin{array}{ll}
\alpha_{k p t}^{R}=\alpha_{k p, t-1}^{R}+\sum_{j=1}^{J} \sum_{v=1}^{V} d a_{j k p v t}-d t_{k p t} & \forall k, p, t \\
\beta_{k c t}^{R}=\beta_{k c, t-1}^{R}+\sum_{p=1}^{P} B O C_{p c} d t_{k p t}-d_{k c t}-\sum_{i=1}^{I} \sum_{v=1}^{V} a f_{k i c v t} & \forall k, c, t
\end{array}
$$




$$
\begin{array}{lc}
\sum_{p=1}^{P} B O C_{p c} \cdot d t_{k p t} \cdot\left(1-\theta_{k c t}\right) \leq d_{k c t} & \forall k, c, t \\
\alpha_{k p t}^{R} \leq \bar{\alpha}_{k p}^{R} & \forall k, p, t \\
\beta_{k c t}^{R} \leq \bar{\beta}_{k c}^{R} & \forall k, c, t \\
d t_{k p t} \leq M D T_{k p}^{R} \cdot \sigma_{k p t} & \forall k, p, t \\
\sum_{k=1}^{K} \sum_{i=1}^{I} \sum_{c=1}^{C} \sum_{t=1}^{T} a f_{k i c v t} \leq c a p_{v}^{R F} & \forall v \\
\sum_{k=1}^{K} \sum_{i=1}^{I} \sum_{c=1}^{C} \sum_{v=1}^{V} d i s_{k i}^{R F} . E m i s_{k i c v t}^{R F} \cdot a f_{k i c v t} \leq T E_{t}^{\max R F} & \forall t \\
a f_{k i c v t}, d a_{j k p t}, d t_{k p t}, d_{k c t}, \alpha_{k p t}^{R}, \beta_{k c t}^{R} \geq 0 \& \in Z & \forall k, i, p, c, v, t \\
\sigma_{k p t} \in\{0,1\} & \forall k, p, t
\end{array}
$$

Expression (1) maximizes the profit of the recycling centers. The profit is equal to revenue minus total costs, including recycling, disassembly, testing, disposal, inventory, and transportation costs. The objective function (2) minimizes the amount of greenhouse gas emissions of each vehicle per kilometer for a unit of eligible components between the recycling centers and remanufacturing/manufacturing factories. Constraint sets (3) and (4) are the inventory equations for returned products and eligible components. Constraint set (5) shows the quantity constraint for disposed of components. Constraint sets (6) and (7) ensure that the inventory rate of returned products and eligible components do not exceed the maximum level. Constraint set (8) represents that the quantity of returned products to be disassembled and tested in a recycling center does not exceed the maximum amount. Constraint set (9) guarantees that the quantity of eligible components that are transported by a vehicle from the recycling centers to the remanufacturing/manufacturing factories does not exceed the capacity of the vehicle. Constraint set (10) ensures the sum of greenhouse gas emissions for the transportation system between recycling centers and factories does not exceed the maximum allowance. Constraint sets (11) and (12) describe the value ranges of the variables.

\section{3-2- The manufacturing/remanufacturing centers, the second level}

The bi-objective MIP model at the second level (SLM) to maximize profit and minimize adverse environmental effects is as follows: 


$$
\begin{aligned}
& \max F_{1}^{F}=\sum_{i=1}^{I} \sum_{j=1}^{J} \sum_{p=1}^{P} \sum_{v=1}^{V} \sum_{t=1}^{T} M P N_{i j p t} f d n_{i j p v t}+\sum_{i=1}^{I} \sum_{j=1}^{J} \sum_{p=1}^{P} \sum_{v=1}^{V} \sum_{t=1}^{T} M P R_{i j p t} f d r_{i j p v t}- \\
& \left\{\sum_{i=1}^{I} \sum_{p=1}^{P} \sum_{t=1}^{T}\left(S A_{i p t} . \eta_{i p t}+U A C_{i p t} x_{i p t}\right)+\sum_{i=1}^{I} \sum_{c=1}^{C} \sum_{t=1}^{T}\left(S P_{i c t} . \pi_{i c t}+U P C_{i c t} w_{i c t}\right)+\right. \\
& \sum_{i=1}^{I} \sum_{p=1}^{P} \sum_{t=1}^{T}\left(S R A_{i p t} \cdot \delta_{i p t}+U R A C_{i p t} \cdot y_{i p t}\right)+\sum_{c=1}^{C}\left(S R P_{i c t} \cdot \tau_{i c t}+U R P C_{i c t} \cdot z_{i c t}\right)+ \\
& \sum_{i=1}^{I} \sum_{p=1}^{P} \sum_{t=1}^{T}\left(I C N P_{i p t}^{F} \cdot \lambda_{i p t}^{F}+I C R M P_{i p t}^{F} \cdot x_{i p t}^{F}\right)+\sum_{i=1}^{I} \sum_{c=1}^{C} \sum_{t=1}^{T}\left(I C Q C_{i c t}^{F} \cdot \beta_{i c t}^{F}+I C N C_{i c t}^{F} \cdot \zeta_{i c t}^{F}+I C R C_{i c t}^{F} \cdot \xi_{i c t}^{F}\right)+ \\
& \left.\sum_{i=1}^{I} \sum_{j=1}^{J} \sum_{p=1}^{P} \sum_{v=1}^{V} \sum_{t=1}^{T}\left(U T C_{i j p v t}^{F D} f d n_{i j p v t}+U T C_{i j p v t}^{F D} f d r_{i j p v t}\right)+\sum_{k=1}^{K} \sum_{i=1}^{I} \sum_{c=1}^{C} \sum_{v=1}^{V} \sum_{t=1}^{T} P P C_{k i c t} . a f_{k i c v t}\right\} \\
& \min F_{2}^{F}=\sum_{i=1}^{I} \sum_{j=1}^{J} \sum_{p=1}^{P} \sum_{v=1}^{V} \sum_{t=1}^{T} \operatorname{dis}_{i j}^{F D} \operatorname{Emis}_{i j p v t}^{F D} .\left(f d n_{i j p v t}+f d r_{i j p v t}\right)+\sum_{i=1}^{I} \sum_{c=1}^{C} \sum_{t=1}^{T} \operatorname{Emispnc}_{i c t}^{F} w_{i c t}+\sum_{i=1}^{I} \sum_{c=1}^{C} \sum_{t=1}^{T} \operatorname{Emisprc}_{i c t}^{F} \cdot z_{i c t}
\end{aligned}
$$

s.t.

$$
\begin{array}{rlrl}
\beta_{i c t}^{F} & =\beta_{i c, t-1}^{F}+\sum_{k=1}^{K} \sum_{v=1}^{V} a f_{k i c v t}-z_{i c t} & & \forall i, c, t \\
\zeta_{i c t}^{F} & =\zeta_{i c, t-1}^{F}+W_{i c t}-\sum_{p=1}^{P} B O C_{p c} x_{i p t} & & \forall i, c, t \\
\xi_{i c t}^{F} & =\xi_{i c, t-1}^{F}+z_{i c t}-\sum_{p=1}^{P} B O C_{p c} \cdot y_{i p t} & \\
\lambda_{i p t}^{F}=\lambda_{i p, t-1}^{F}+x_{i p t}-\sum_{j=1}^{J} \sum_{v=1}^{V} f d n_{i j p v t} & \forall i, c, t \\
x_{i p t}^{F}=x_{i p, t-1}^{F}+y_{i p t}-\sum_{j=1}^{J} \sum_{v=1}^{V} f d r_{i j p v t} & \\
\beta_{i c t}^{F} \leq \bar{\beta}_{i c}^{F} & \forall i, p, t \\
\zeta_{i c t}^{F} & \leq \bar{\zeta}_{i c}^{F} & \\
\xi_{i c t}^{F} & \leq \bar{\xi}_{i c}^{F} & \forall i, p, t \\
\lambda_{i p t}^{F} \leq \bar{\lambda}_{i p}^{F} & \forall i, c, t \\
x_{i p t}^{F} \leq \bar{x}_{i p}^{F} & \forall i, c, t \\
w_{i c t} \leq M P_{i c} . \pi_{i c t} & \forall i, c, t \\
z_{i c t} \leq M R P_{i c} \cdot \tau_{i c t} & \forall i, p, t \\
x_{i p t} \leq M A_{i p} \eta_{i p t} & \forall i, p, t \\
y_{i p t} \leq M R A_{i p} \delta_{i p t} & \forall i, c, t \\
& & \forall i, c, t \\
& & \forall i, p, t \\
& & \forall i, p, t
\end{array}
$$




$$
\begin{aligned}
& \sum_{i=1}^{I} \sum_{j=1}^{J} \sum_{p=1}^{P} \sum_{t=1}^{T}\left(f d n_{i j p v t}+f d r_{i j p v t}\right) \leq \operatorname{cap}_{v}^{F D} \quad \forall v \\
& \sum_{i=1}^{I} \sum_{j=1}^{J} \sum_{p=1}^{P} \sum_{v=1}^{V} d i s_{i j}^{F D} \cdot \text { Emis }_{i j p v t}^{F D} \cdot\left(f d n_{i j p v t}+f d r_{i j p v t}\right) \leq T E_{t}^{\max F D} \quad \forall t \\
& \sum_{i=1}^{I} \sum_{c=1}^{C} \text { Emispnc }_{i c t}^{F} w_{i c t}+\sum_{i=1}^{I} \sum_{c=1}^{C} \text { Emisprc }_{i c t}^{F} z_{i c t} \leq O P E_{t}^{\max F} \quad \forall t \\
& f d n_{i j p v t}, f d r_{i j p v t}, a f_{k i c v t}, x_{i p t}, y_{i p t}, w_{i c t}, z_{i c t}, \beta_{i c t}^{F}, \zeta_{i c t}^{F}, \xi_{i c t}^{F}, \lambda_{i p t}^{F}, x_{i p t}^{F} \geq 0 \& \in Z \quad \forall i, j, p, c, v, t \\
& \pi_{i c t}, \tau_{i c t}, \eta_{i p t}, \delta_{i p t} \in(0,1) \\
& \forall i, c, p, t
\end{aligned}
$$

Expression (13) maximizes the profit of the remanufacturing/manufacturing factories. The profit is equal to sales revenue minus total costs, including manufacturing, remanufacturing, inventory, purchase, and transportation costs. The objective function (14) minimizes the amount of greenhouse gas emissions of each vehicle per kilometer for a unit of new products and remanufactured products between remanufacturing/manufacturing factories and distribution centers, as well as the amount of greenhouse gas emissions during processing and reprocessing. Constraint sets (15)-(19) represent the inventory equations for eligible, new, remanufactured components, and products. Constraint sets (20)-(24) ensure that the inventory quantities of eligible, new, remanufactured components and products do not exceed the maximum quantity. Constraint sets (25)-(28) guaranty that the quantity of processed and reprocessed components, as well as the quantity of new and remanufactured products, do not exceed the capacity of relevant factories. Constraint set (29) considers that the amount of new and remanufactured products that are transported by a vehicle from the manufacturing/remanufacturing factories to distribution centers does not exceed the capacity of the vehicle. Constraint set (30) ensures the sum of greenhouse gas emissions for the transportation system between factories and distributions centers does not exceed the maximum allowance. Constraint set (31) represents that the sum of greenhouse gas emissions for processing and reprocessing operations in factories must be less than the maximum allowance. Constraint sets (32) and (33) describe the value ranges of the variables.

\section{3-3- The Distribution centers, the third level}

The bi-objective MIP model at the third level (TLM) to maximize profit and minimize adverse environmental effects is as bellows: 
$\max F_{1}^{D}=\sum_{j=1}^{J} \sum_{p=1}^{P} \sum_{t=1}^{T} S P N_{j p t} .\left(D N M_{j p t}-n s S_{j p t}\right)+\sum_{j=1}^{J} \sum_{p=1}^{P} \sum_{t=1}^{T} S P R_{j p t} .\left(D R M_{j p t}-r s S_{j p t}\right)+$

$\sum_{j=1}^{J} \sum_{k=1}^{K} \sum_{p=1}^{P} \sum_{v=1}^{V} \sum_{t=1}^{T} U R C C_{j k p t} . d a_{j k p v t}-\left\{\sum_{i=1}^{I} \sum_{j=1}^{J} \sum_{p=1}^{P} \sum_{v=1}^{V} \sum_{t=1}^{T}\left(M P N_{i j p t} f d n_{i j p v t}+M R N_{i j p t} f d r_{i j p v t}\right)+\right.$

$\sum_{j=1}^{J} \sum_{p=1}^{P} \sum_{t=1}^{T} U R C D_{j p t} \cdot \gamma_{j p t}+\sum_{j=1}^{J} \sum_{p=1}^{P} \sum_{t=1}^{T}\left(U S N P_{j p t} . n s s_{j p t}+U S R P_{j p t} . r s s_{j p t}\right)+$

$\left.\sum_{j=1}^{J} \sum_{p=1}^{P} \sum_{t=1}^{T}\left(I C N P_{j p t}^{D} \cdot \lambda_{j p t}^{D}+I C R M P_{j p t}^{D} \cdot x_{j p t}^{D}+I C R P_{j p t}^{d} \cdot \alpha_{j p t}^{D}\right)+\sum_{j=1}^{J} \sum_{k=1}^{K} \sum_{p=1}^{P} \sum_{v=1}^{V} \sum_{t=1}^{T} U T C_{j k p v t}^{D R} \cdot d a_{j k p v t}\right\}$

$\min F_{2}^{D}=\sum_{j=1}^{J} \sum_{k=1}^{K} \sum_{p=1}^{P} \sum_{v=1}^{V} \sum_{t=1}^{T} d i s_{j k}^{D R} \operatorname{Emis}_{j k p v t}^{D R} d a_{j k p v t}$

s.t.

$\lambda_{j p t}^{D}=\lambda_{j p, t-1}^{D}+\sum_{i=1}^{I} \sum_{v=1}^{V} f d n_{i j p v t}-\left(D N M_{j p t}-n s s_{j p t}\right) \quad \forall j, p, t$

$x_{j p t}^{D}=x_{j p, t-1}^{D}+\sum_{i=1}^{I} \sum_{v=1}^{V} f d r_{i j p v t}-\left(D R M_{j p t}-r s s_{j p t}\right)$

$\forall j, p, t$

$\alpha_{j p t}^{D}=\alpha_{j p, t-1}^{D}+\gamma_{j p t}-\sum_{k=1}^{K} \sum_{v=1}^{V} d a_{k j p v t}$

$\forall j, p, t$

$\gamma_{j p t} \leq E P A_{j p t}$

$\forall j, p, t$

$n s s_{j p t} \leq D N M_{j p t}$

$\forall j, p, t$

$r s s_{j p t} \leq D R M_{j p t}$

$\forall j, p, t$

$\lambda_{j p t}^{D} \leq \bar{\lambda}_{j p}^{D}$

$\forall j, p, t$

$x_{j p t}^{D} \leq \bar{x}_{j p}^{D}$

$\forall j, p, t$

$\alpha_{j p t}^{D} \leq \bar{\alpha}_{j p}^{D}$

$\forall j, p, t$

$\sum_{j=1}^{J} \sum_{k=1}^{K} \sum_{p=1}^{P} \sum_{t=1}^{T} d a_{j k p v t} \leq \operatorname{cap}_{v}^{D R}$

$\forall v$

$\sum_{j=1}^{J} \sum_{k=1}^{K} \sum_{p=1}^{P} \sum_{v=1}^{V} d i s_{j k}^{D R} \cdot \operatorname{Emis}_{j k p v t}^{D R} d a_{j k p v t} \leq T E_{t}^{\max D R}$

$\forall t$

$n s s_{j p t}, r s s_{j p t}, d a_{j k p v t}, f d n_{i j p v t}, f d r_{i j p v t}, \gamma_{j p t}, \lambda_{j p t}^{D}, x_{j p t}^{D}, \alpha_{j p t}^{D} \geq 0 \& \in Z \quad \forall i, j, p, v, t$

Expression (34) maximizes the profit of the distribution centers. The profit is equal to sales revenue minus total costs, including the purchase of new and returned products, shortage, inventory, and transportation costs. The objective function (35) minimizes the amount of greenhouse gas emissions of each vehicle per kilometer for a unit of returned product between distribution and recycling centers. Constraint sets (36)-(38) show the inventory equations for new, remanufactured, and returned products. Constraint set (39) ensures that the number of collected returned products does not exceed the number of returned products available in the 
market. Constraint sets (40) and (41) represent the quantity shortage for new and remanufactured products. Constraint sets (42)-(44) guaranty that the inventory rate of remanufactured, new, and returned products do not exceed the maximum level. Constraint set (45) ensures that the quantity of returned products, which are transported by a vehicle from distribution centers to recycling centers, does not overstep the capacity of the vehicle. Constraint set (46) guarantees that the sum of greenhouse gas emissions for the transportation system between distribution and recycling centers does not exceed the maximum allowance. Constraint set (47) defines the value ranges of the variables.

\section{4- Solution approach}

The multi-level linear programming is NP-hard (Hansen et al. 1992), so finding its solutions are usually hard. In this paper, the proposed three-level bi-objective programming formulation is solved based on a hierarchical iterative approach using the LP-metric method for small-size instances and the NSGA-II algorithm for large-size instances, respectively. Moreover, the NSGA-II algorithm is combined with MC simulation (MCNSGA-II) to tackle uncertainty in demand.

\section{4-1- LP-metric method}

LP-metric is a classical approach which is applied to solve multi-objective models. The method attempts to find the best solution, which has the shortest distance from the ideal solution. Therefore, the LP-metric function (48) is applied to compute the distance of an available solution to the ideal solution (Pasandideh et al. 2015).

$$
L p=\left\{\sum_{j=1}^{k} w_{j} \times\left[\frac{f_{j}\left(x^{* j}\right)-f_{j}(x)}{f_{j}\left(x^{* j}\right)-f_{j}\left(\bar{x}^{j}\right)}\right]^{p}\right\}^{\frac{1}{p}}
$$

where $f_{j}(x)$ and $w_{j}$ represent the $j^{\text {th }}$ objective function and its importance degree, respectively. $p$ demonstrates the emphasis degree on available deviations. To calculate the ideal value of the $j^{\text {th }}$ objective function $\left(f_{j}\left(x^{* j}\right)\right)$, the model with the $j^{\text {th }}$ objective function and the existing constraints is solved. Moreover, to find the anti-ideal value of the $j^{\text {th }}$ objective function ( $\left.f_{j}\left(\bar{x}^{j}\right)\right)$, the objective function is reversed. Finally, we minimize the LP-metric function (48) subject to the constraints to obtain the solution.

\section{4-2- The NSGA-II algorithm}

In this paper, the NSGA-II algorithm (Deb et al. 2002) is applied for solving the considered bi-objective models at each level. Note that the algorithm is adopted to solve medium and largescale instances.

\section{4-2-1- Encoding}

Regarding the characteristic of the FLM, $\left(a f_{k i c v t}, d t_{k p t}, d_{k c t}\right)$ and $\left(\alpha_{k p t}^{R}, \beta_{k c t}^{R}, \sigma_{k p t}\right)$ are considered as the decision variables and the state variables, respectively. To encoding the 
solution of the FLM, each decision variable is a part of the chromosome, that generates chromosomes $C_{m=1}^{n}=\left(a f_{k i c v t}, d t_{k p t}, d_{k c t}\right)_{m=1}^{n} . n=\{1,2, \ldots, N p o p\}$ indicates the index of population size, Npop is the population size, $m=\{1,2, \ldots$, Maxiter $\}$ indicates the index of generation, Maxiter is the maximum number of the generation.

Accordingly, by considering $\left(f d n_{i j p v t}, f d r_{i j p v t}, x_{i p t}, y_{i p t}, w_{i c t}, z_{i c t}, s u b c_{i c t}\right)$ as the decision variables, and $\left(\beta_{i c t}^{F}, \zeta_{i c t}^{F}, \xi_{i c t}^{F}, \pi_{i c t}, \tau_{i c t}, \eta_{i p t}, \delta_{i p t}\right)$ as the state variables in the SLM as well as $\left(n s s_{j p t}, r s s_{j p t}, d a_{j k p v t}, \gamma_{j p t}\right)$ as the decision variables, and $\left(\lambda_{j p t}^{D}, x_{j p t}^{D}, \alpha_{j p t}^{D}\right)$ as the state variables in the TLM, the chromosomes of the levels are defined similarly to the FLM. Due to the suggested model, the values of decision variables in the chromosomes are randomly generated based on the introduced intervals in Table 2- Table 4.

Table 2: Generation interval of decision variables for the FLM

\begin{tabular}{cc}
\hline Variables & Generation interval \\
\hline$a f_{k i c v t}$ & unif $\left[0, \min \left(\operatorname{cap}_{v}^{R F}, \frac{T E_{t}^{\max R F}}{d i s_{k i}^{R F} \cdot E m i s_{k i c v t}^{R F}}\right)\right]$ \\
$d t_{k p t}$ & unif $\left[0, M D T_{k p}^{R}\right]$ \\
$d_{k c t}$ & unif $\left[0, \operatorname{round}\left(\left(1-\theta_{k c t}\right) d t_{k p t}\right)\right]$ \\
\hline
\end{tabular}

Table 3: Generation interval of decision variables for the SLM

\begin{tabular}{cc}
\hline Variables & Generation interval \\
\hline$f d n_{i j p v t}, f d r_{i j p v t}$ & unif $\left[0, \min \left(\frac{c a p_{v}^{F D}}{2}, \frac{T E_{t}^{\max F D}}{2 \cdot d i s_{i j}^{F D} \cdot E m i s_{i j p v t}^{F D}}\right)\right]$ \\
$x_{i p t}$ & unif $\left[0, M A_{i p}\right]$ \\
$y_{i p t}$ & unif $\left[0, M R A_{i p}\right]$ \\
$w_{i c t}$ & unif $\left[0, \min \left(M P_{i c}, \frac{O P E_{t}^{\max F}}{2 \cdot E m i s p n c_{i c t}^{F}}\right)\right]$ \\
$z_{i c t}$ & unif $\left[0, \min \left(M R P_{i c}, \frac{O P E_{t}^{\max F}}{2 \cdot E m i s p r c_{i c t}^{F}}\right)\right]$ \\
$s u b c_{i c t}$ & unif $\left[0, w_{i c t}\right]$ \\
\hline
\end{tabular}

Table 4: Generation interval of decision variables for the TLM

\begin{tabular}{cc}
\hline Variables & Generation interval \\
\hline$n s s_{j p t}$ & unif $\left[0, D N M_{j p t}\right]$ \\
$r s s_{j p t}$ & unif $\left[0, D R M_{j p t}\right]$
\end{tabular}




$$
\begin{array}{cc}
d a_{j k p v t} & \text { unif }\left[0, \min \left(c a p_{v}^{D R}, \frac{T E_{t}^{\max D R}}{d i s_{j k}^{D R} \cdot E m i s_{j k p v t}^{D R}}\right)\right] \\
\gamma_{j p t} & \text { unif }\left[0, E P A_{j p t}\right]
\end{array}
$$

\section{4-2-2- Crossover}

The crossover operator is used to transfer the same characteristics from parents to nextgeneration children. The simplest types of crossovers are single-point, two-point, and uniform crossover. In this paper, the two-point crossover is applied. First, two parents are selected, and then two numbers are randomly selected to determine the cut-off area. The first child inherits the cut region from the second parent, and the second child inherits it from the first parent. Figure 2 shows the crossover operator in this study.

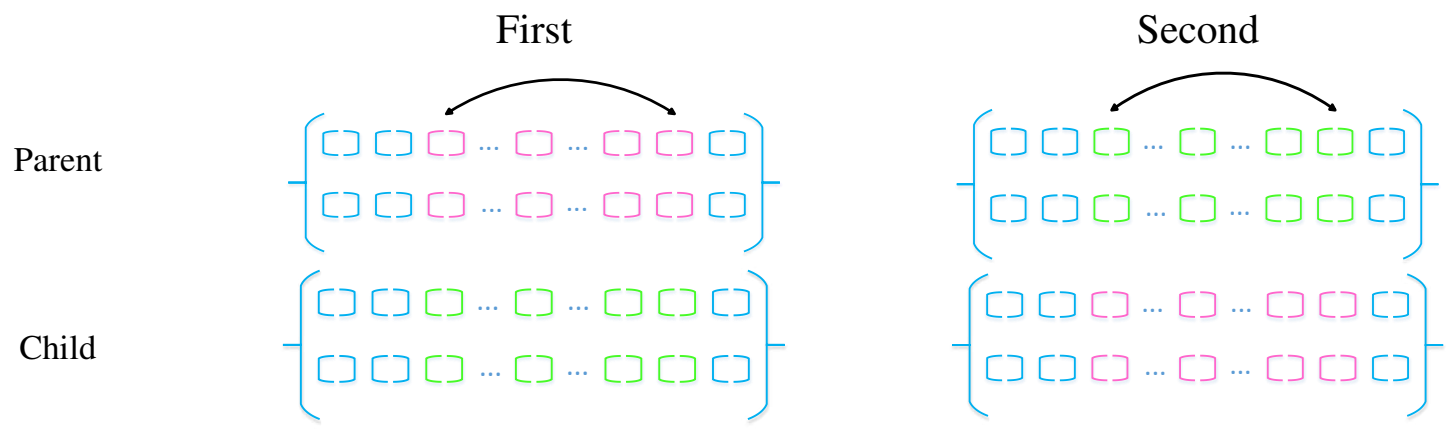

Figure 2: The crossover operator

\section{4-2-3- Mutation}

The mutation operator is usually performed with a very low probability. In the present study, two chromosomes from the parents are randomly selected, and the position of the two chromosomes is changed, as shown in Figure 3.

Parent

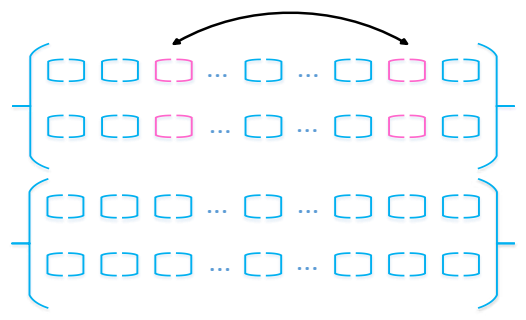

Figure 3: The mutation operator

\section{4-3- The hierarchical iterative approach}

The proposed three-level model is solved based on a hierarchical iterative algorithm. The description of the algorithm is as below:

Step 1: Determine the initial value of variable $d a_{j k p v t}$. It is randomly generated from formula (45) and (46) at iteration 0 ( Iter $=0)$.

Step 2: Solve the FLM using the values of $d a_{j k p v t}$. Note that the values of $d a_{j k p v t}$ are from Step 1 and Step 4 in the $(\text { Iter }-1)^{\text {th }}$ iteration when Iter $=0$ and Iter $>0$, respectively. The LPmetric method is used to solve the FLM and obtain a solution set 
$\left(a f_{k i c v t}, d t_{k p t}, d_{k c t}, \alpha_{k p t}^{R}, \beta_{k c t}^{R}, \sigma_{k p t}\right)^{\text {Iter }}$ and its LP-metric function value ( $\left.F_{1}^{\text {Iter }}\right)$. The values of $a f_{k i c v t}$ will be considered for the SLM as a parameter.

Step 3: Solve the SLM using the values $a f_{\text {kicvt }}$ from Step 2. The LP-metric method is applied to solve SLM and obtain a solution set $\left(f d n_{i j p v t}, f d r_{i j p v t}, x_{i p t}, y_{i p t}, w_{i c t}, z_{i c t}, s u b c_{i c t}, \beta_{i c t}^{F}, \zeta_{i c t}^{F}, \xi_{i c t}^{F}, \pi_{i c t}, \tau_{i c t}, \eta_{i p t}, \delta_{i p t}\right)^{I t e r}$ and its LP-metric function value $\left(F_{2}^{\text {Iter }}\right.$ ), in which the values $f d n_{i j p v t}$ and $f d r_{i j p v t}$ will be considered for the TLM as parameters.

Step 4: Solve the TLM applying the values of $f d n_{i j p v t}$ and $f d r_{i j p v t}$ from Step 3. The LP-metric method is used to solve the TLM and obtain a solution set $\left(n s s_{j p t}, r s s_{j p t}, d a_{j k p v t}, \gamma_{j p t}, \lambda_{j p t}^{D}, x_{j p t}^{D}, \alpha_{j p t}^{D}\right)^{\text {Iter }}$ and its LP-metric function value $\left(F_{3}^{\text {Iter }}\right)$, in which the values of $d a_{j k p v t}$ will be considered for the FLM as a parameter in a conceivable new iteration.

Step 5: Check that the stopping condition has been met, that is:

$\max \left(\overline{w_{1}}, \overline{w_{2}}, \overline{w_{3}}\right) \leq w^{*}$

$\overline{w_{1}}=\left|\frac{F_{1}^{\text {Iter }+1}-F_{1}^{\text {Iter }}}{F_{1}^{\text {Iter }+1}}\right|, \overline{w_{2}}=\left|\frac{F_{2}^{\text {Iter }+1}-F_{2}^{\text {Iter }}}{F_{2}^{\text {Iter }+1}}\right|, \overline{w_{3}}=\left|\frac{F_{3}^{\text {Iter }+1}-F_{3}^{\text {Iter }}}{F_{3}^{\text {Iter }+1}}\right|$

where $w^{*}$ is an iteration accuracy given in advanced. If so, stop iteration; if not, start Step 2.

Note that the NSGA-II algorithm is implemented in the context of the above procedure. The NSGA-II is a population-based algorithm, so the Mean Ideal Distance (MID) criterion is applied to calculate $\overline{\mathrm{w}}_{1}, \overline{\mathrm{w}}_{2}$, and $\overline{\mathrm{w}}_{3}$. By considering the equation (51) to calculate MID, the equation (52) is used to calculated $\overline{\mathrm{w}}_{1}, \overline{\mathrm{w}}_{2}$, and $\overline{\mathrm{w}}_{3}$ in Step 5.

$$
\begin{aligned}
& M I D_{l}=\frac{\sum_{n=1}^{N_{l}} \sqrt{\left(\frac{f 1_{\text {ln }}-f 1_{l_{-} \text {best }}}{f 1_{l_{-} \max }-f 1_{l_{-} \min }}\right)^{2}+\left(\frac{f 2_{\text {ln }}-f 2_{l_{-} \text {best }}}{f 2_{l_{-} \max }-f 2_{l_{-} \min }}\right)^{2}}}{N_{l}}, \quad \forall \mathrm{l}=1,2,3 \\
& \bar{w}_{1}=\left|\frac{M I D_{1}^{\text {Iter }+1}-M I D_{1}^{\text {Iter }}}{M I D_{1}^{\text {Iter }+1}}\right|, \quad \bar{w}_{2}=\left|\frac{M I D_{2}^{\text {Iter }+1}-M I D_{2}^{\text {Iter }}}{M I D_{2}^{\text {Iter }+1}}\right|, \bar{w}_{3}=\left|\frac{M I D_{3}^{\text {Iter }+1}-M I D_{3}^{\text {Iter }}}{M I D_{3}^{\text {Iter }+1}}\right|
\end{aligned}
$$

where $f 1_{l_{-} \text {best }}$ and $f 2_{l_{-} \text {best }}$ are the ideal point of the first and the second objective functions at level $l . f 1_{l_{-} \max }$ and $f 2_{l_{-} \max }$ are defined as the largest value of the two objective functions in the obtained non-dominated solutions at level $l . f 1_{l_{-} \min }$ and $f 2_{l_{-} \min }$ are defined as the smallest value of the two objective functions in the obtained non-dominated solutions at level $l . f 1_{\ln }$ and $f 2_{\text {ln }}$ are the value of the two objective functions of the $n^{\text {th }}$ non-dominated solution at level $l$. $N_{l}$ indicates the number of the non-dominated solutions at level $l$.

\section{4-4- Monte Carlo Simulation}

MC simulation is premier to a deterministic simulation of a system when the input variables of the system are random. In this simulation approach, a single value is selected for 
each input random variable based on the best guess by the modeler. The approach is then run, and the output is considered. This output is a single value or a single set of values based on the selected input. MC simulation randomly samples values from every input variable distribution and applies the sample to compute the model's output. This procedure is iterated many times until the modeler achieves a sense of how the output changes given the random input values (Sokolowski and Banks 2010).

In this paper, it is assumed that the demand parameter in the third level (distribution centers) is uncertain. We use MC simulation to deal with the uncertainty in the NSGA-II algorithm. Therefore, MC simulation is applied to appraise the objective functions of each chromosome, considering the probability distribution of the demand that is obtained from historical data. Moreover, the expected value of each objective function related to a chromosome is estimated by simulation. Moreover, we calculate the number of simulation iterations using equation (53).

$$
\Omega \geq\left[z_{\alpha / 2} \cdot \frac{S}{E}\right]^{2}
$$

where $\Omega$ is the number of iteration, $E$ is the desired margin of error, and $S$ denotes the computed standard deviation of the output. Furthermore, $z_{\alpha / 2}$ shows the critical value of the normal distribution for $\alpha / 2$ (Brandimarte 2014).

Figure 4 displays the flowchart of the MCNSGA-II algorithm to generate a Pareto set in the third level. 


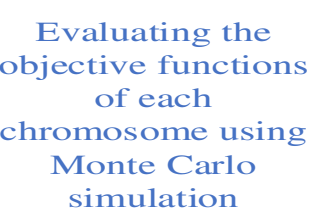

simulation

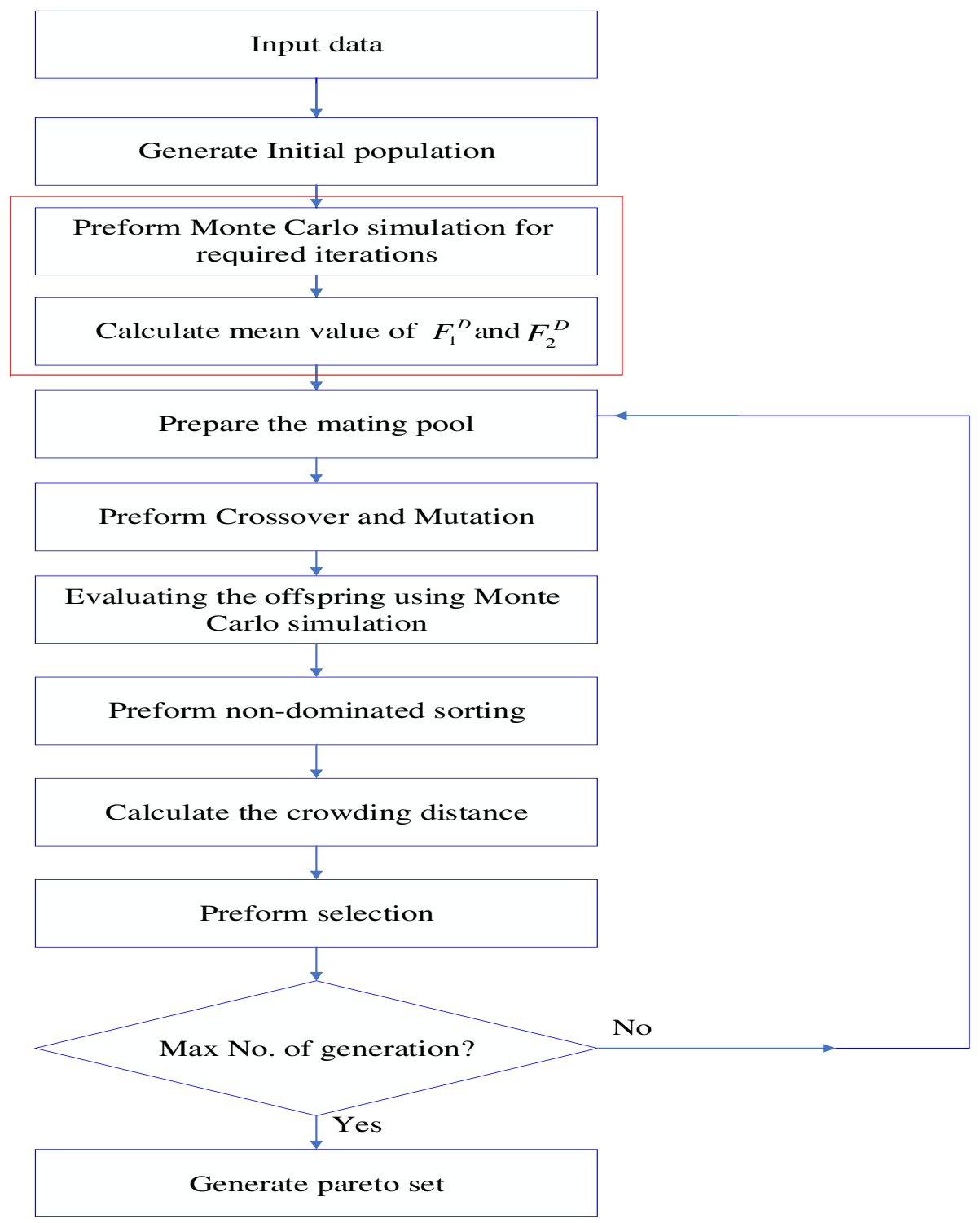

Figure 4: The flowchart of the MCNSGA-II algorithm in the third level

\section{4-5- Comparison of multi-objective solution methods}

Two metrics, set coverage and spacing, are usually applied to evaluate convergence and diversity of a multi-objective solution method. We apply the following metrics to assess the obtained non-dominated solutions the NSGA-II and MCNSGA-II algorithms.

\section{4-5-1- Set coverage metric}

Zitzler and Thiele (1999) proposed the set coverage metric $C(A, B)$ that computes the ratio of solutions in $B$ are weakly dominated by solution $A$. 


$$
C(A, B)=\frac{|\{b \in B \mid \exists a \in A: a \leq b\}|}{|B|}
$$

where, $C(A, B)=1$ means that all members of $B$ are weakly dominated by $A . C(A, B)=0$ shows that no member of $B$ is weakly dominated by $A$.

\section{4-5-2- Spacing metric}

Scott (1995) presented the following metric to calculate the comparative distance between successive solutions in the non-dominated set $(Q)$.

$$
S=\sqrt{\frac{1}{Q} \sum_{i=1}^{|Q|}\left(d_{i}-\bar{d}\right)^{2}}
$$

where, $d_{i}=\min _{k \in Q \wedge k \neq i} \sum_{m=1}^{M}\left|f_{m}^{i}-f_{m}^{k}\right|, M$ indicates the number of objective functions, $f_{m}^{i}$ and $f_{m}{ }_{m}$ are the $i^{\text {th }}$ and $k^{\text {th }}$ value of the objective function $m$ in set $Q$, respectively, and $\bar{d}=\sum_{i=1}^{|Q|} d_{i} /|Q|$. The metric computes the standard deviations of different values; thus, the small value of the metric demonstrates that the solutions are approximately uniformly spaced.

Based on the above-mentioned metrics, an algorithm with a greater $C$ and a smaller $S$ is preferred.

\section{5- Computational results}

In this section, numerical experiments are conducted to investigate the performance of the three-level bi-objective MIP model, the NSGA-II algorithm, and the MCNSGA-II algorithm based on random data. First, we present how the test problems are generated. The Taguchi approach is then employed to tune the parameters of the NSGA-II algorithm, and the best level of each parameter is obtained by the signal-to-noise diagram in the Minitab software. Sensitivity analysis is then performed for different weights of LP-metric function, and the best weight for each level is selected. The results obtained by the LP-metric method and the NSGA II algorithm are compared, and the relative distance of each sample is determined. Note that the LP-metric method and the NSGA II algorithm are implemented in GAMS and MATLAB, respectively, and are tested on a computer with a $2.3 \mathrm{GHz}$ CPU and 8.0 GB of RAM. Finally, the MCNSGA-II algorithm is implemented on two instances, and the results are compared with the NSGA-II algorithm.

\section{5-1- Data generation}

We need to test the suggested mathematical model and the solution approach using randomly generated test problems. The dimension of the test problems that are categorized into small, medium, and large-scale are introduced in Table 5. 
Table 5: Dimension of the test problems

\begin{tabular}{lccc}
\hline \multicolumn{1}{c}{ Index } & \multicolumn{2}{c}{ Level } & \\
& Small & medium & Large \\
\hline Number of recycling centers $(k)$ & {$[2,4]$} & {$[5,7]$} & $\geq 8$ \\
Number of manufacture/remanufacture factories $(i)$ & {$[2,4]$} & {$[5,7]$} & $\geq 8$ \\
Number of distributions $(j)$ & {$[2,5]$} & {$[6,8]$} & $\geq 9$ \\
Number of components $(c)$ & {$[2,6]$} & {$[7,9]$} & $\geq 10$ \\
Number of products $(p)$ & {$[2,3]$} & {$[4,6]$} & $\geq 7$ \\
Number of vehicles $(v)$ & {$[2,4]$} & {$[6,7]$} & $\geq 8$ \\
Number of periods $(t)$ & {$[2,7]$} & {$[8,12]$} & $\geq 12$ \\
\hline
\end{tabular}

Moreover, the values of model parameters are randomly generated based on a uniform distribution that are shown in Table 6.

Table 6: The values of model parameters

\begin{tabular}{|c|c|c|}
\hline $\begin{array}{c}\text { First level parameters } \\
\end{array}$ & Second level parameters & Third level parameters \\
\hline$P P C_{\text {kict }}=$ Uniform $(200,400)$ & $S A_{i p t}=$ Uniform $(60,70)$ & $S P N_{j p t}=\operatorname{Uniform}(20000,30000)$ \\
\hline$U R C C_{j k p t}=$ Uniform $(100,200)$ & $S R A_{i p t}=$ Uniform $(40,60)$ & $S P R_{j p t}=\operatorname{Uniform}(10000,20000)$ \\
\hline$S D T_{k p t}=$ Uniform $(50,60)$ & $U A C_{i p t}=$ Uniform $(55,65)$ & $D N M_{j p t}=$ Uniform $(30,50)$ \\
\hline$U D T C_{k p t}=$ Uniform $(45,50)$ & $U R A C_{i p t}=$ Uniform $(45,55)$ & $D R M_{j p t}=$ Uniform $(15,18)$ \\
\hline$U D C_{k c t}=$ Uniform $(20,30)$ & $S P_{i c t}=$ Uniform $(70,80)$ & $U R C D_{j p t}=$ Uniform $(30,60)$ \\
\hline 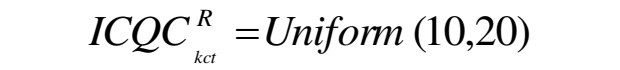 & $S R P_{i c t}=$ Uniform $(65,70)$ & $U S N P_{j p t}=$ Uniform $(53,75)$ \\
\hline$I C R P_{k p t}^{R}=$ Uniform $(10,20)$ & $M P N_{i j p t}=$ Uniform $(700,900)$ & $U S R P_{j p t}=$ Uniform $(33,45)$ \\
\hline$U T C_{\text {kicvt }}^{R F}=$ Uniform $(40,60)$ & $M P R_{i j p t}=$ Uniform $(600,700)$ & $I C N P_{j p t}^{D}=$ Uniform $(17,27)$ \\
\hline$\theta_{k c t}=$ Uniform $(0.85,0.95)$ & $U P C_{i c t}=$ Uniform $(65,75)$ & $\operatorname{ICR} M_{j p t}^{D}=$ Uniform $(16,26)$ \\
\hline $\bar{\alpha}_{k p}^{R}=$ Uniform $(200,250)$ & $U R P C_{i c t}=$ Uniform $(60,70)$ & $I C R P_{j p t}^{D}=$ Uniform $(15,25)$ \\
\hline $\bar{\beta}_{k c}^{R}=$ Uniform $(200,250)$ & $I C N P_{i p t}^{F}=$ Uniform $(15,25)$ & $U T C_{j k p t}^{D R}=\operatorname{Uniform}(50,66)$ \\
\hline$M D T_{k p}^{R}=$ Uniform $(1150,1200)$ & $I C R M P_{i p t}^{F}=$ Uniform $(15,25)$ & $E P A_{j p t}=$ Uniform $(180,200)$ \\
\hline $\operatorname{cap}_{v}^{R F}=$ Uniform $(350,400)$ & $I C Q C_{i c t}^{F}=$ Uniform $(15,25)$ & $\bar{\lambda}_{j p}^{D}=$ Uniform $(100,110)$ \\
\hline dis $_{k i}^{R F}=$ Uniform $(200,300)$ & $I C N C_{i c t}^{F}=$ Uniform $(10,15)$ & $\bar{x}_{j p}^{D}=$ Uniform $(100,110)$ \\
\hline Emis $_{\text {kicvt }}^{R F}=$ Uniform $(0.0001,0.0007)$ & $I C R C_{i c t}^{F}=$ Uniform $(15,25)$ & $\bar{\alpha}_{j p}^{D}=$ Uniform $(100,110)$ \\
\hline$T E_{t}^{\max R F}=$ Uniform $(4000,7000)$ & $U T C_{i j p v t}^{F D}=$ Uniform $(46,63)$ & $\operatorname{cap}_{v}^{D R}=$ Uniform $(350,400)$ \\
\hline
\end{tabular}


Table 6: The values of model parameters

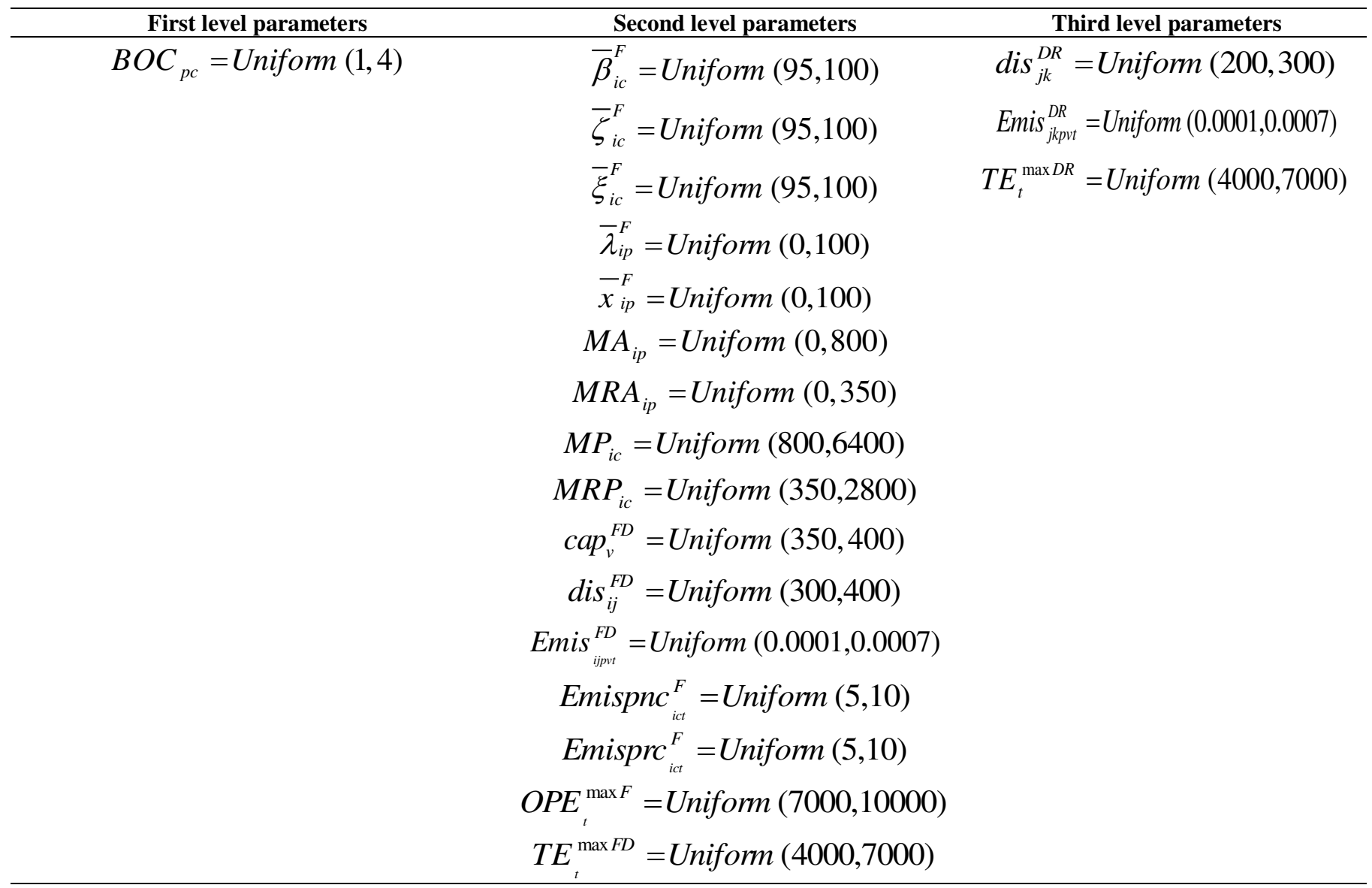

\section{5-2- Parameter tunning of NSGA-II}

The Taguchi experimental design approach is utilized to calibrate the parameters of the NSGA-II algorithm. The approach minimizes the effect of noise and specifies the optimal level of signal factors. To do so, the signal to noise ratio $(\mathrm{S} / \mathrm{N})$, that computes the value of variation of the response, is implemented. Then, the method aims to maximize the $\mathrm{S} / \mathrm{N}$ ratio (Peace 1993). In this paper, MID and maximum scattering (MS) metrics calculate response based on equation (56).

Response $=\frac{M I D}{M S}$

Table 7 exhibits different levels of the factors for NSGA-II algorithm parameters at the recycling centers level to run the Taguchi method.

Table 7: Different levels for NSGA-II algorithm parameters at the recycling centers level

\begin{tabular}{cccccc}
\hline \multirow{2}{*}{ Algorithm } & \multirow{2}{*}{ Parameters } & $\begin{array}{c}\text { Parameter } \\
\text { range }\end{array}$ & $\mathbf{1}$ & $\begin{array}{c}\text { Level } \\
\mathbf{2}\end{array}$ & $\mathbf{3}$ \\
\hline \multirow{3}{*}{ NSGA-II } & Maxiter & {$[100,300]$} & 100 & 200 & 300 \\
& N-pop & {$[50,100]$} & 50 & 75 & 100 \\
& $P c$ & {$[0.85,0.95]$} & 0.85 & 0.9 & 0.95 \\
& $P m$ & {$[0.05,0.15]$} & 0.05 & 0.1 & 0.15 \\
\hline
\end{tabular}


where $P c$ and $P m$ are the crossover and mutation probability, respectively. Then, applying Minitab Software, the L9 design is applied for the NSGA-II algorithm, and experimental results are illustrated in Table 8.

Table 8: The L9 design for the NSGA-II algorithm at the recycling centers level

\begin{tabular}{|c|c|c|c|c|c|c|c|}
\hline \multirow{2}{*}{ Experiment } & \multirow{2}{*}{ Maxiter } & \multirow{2}{*}{ N-pop } & \multirow{2}{*}{ Pc } & \multirow{2}{*}{ Pm } & \multicolumn{3}{|c|}{ Response } \\
\hline & & & & & Small $\left(10^{-7}\right)$ & $\operatorname{Medium}\left(10^{-7}\right)$ & Large $\left(10^{-7}\right)$ \\
\hline 1 & 1 & 1 & 1 & 1 & 3.11793 & 3.30369 & 3.71324 \\
\hline 2 & 1 & 2 & 2 & 2 & 3.47064 & 3.06284 & 3.46838 \\
\hline 3 & 1 & 3 & 3 & 3 & 3.34403 & 3.15414 & 3.20768 \\
\hline 4 & 2 & 1 & 2 & 3 & 3.68890 & 3.75809 & 3.16742 \\
\hline 5 & 2 & 2 & 3 & 1 & 3.18804 & 3.39505 & 3.43168 \\
\hline 6 & 2 & 3 & 1 & 2 & 3.38996 & 2.90125 & 3.37563 \\
\hline 7 & 3 & 1 & 3 & 2 & 3.13996 & 3.46964 & 3.31004 \\
\hline 8 & 3 & 2 & 1 & 3 & 3.13142 & 3.13068 & 3.21475 \\
\hline 9 & 3 & 3 & 2 & 1 & 3.26834 & 3.41561 & 3.08207 \\
\hline
\end{tabular}

The above approach is applied to parameter tuning of the NSGA-II algorithm at the second and the third levels. Figure 5 indicates the effect plot of the $\mathrm{S} / \mathrm{N}$ ratio related to each level. According to Figure 5, the best parameters values of the NSGA-II algorithm for each level are indicated in Table 9.

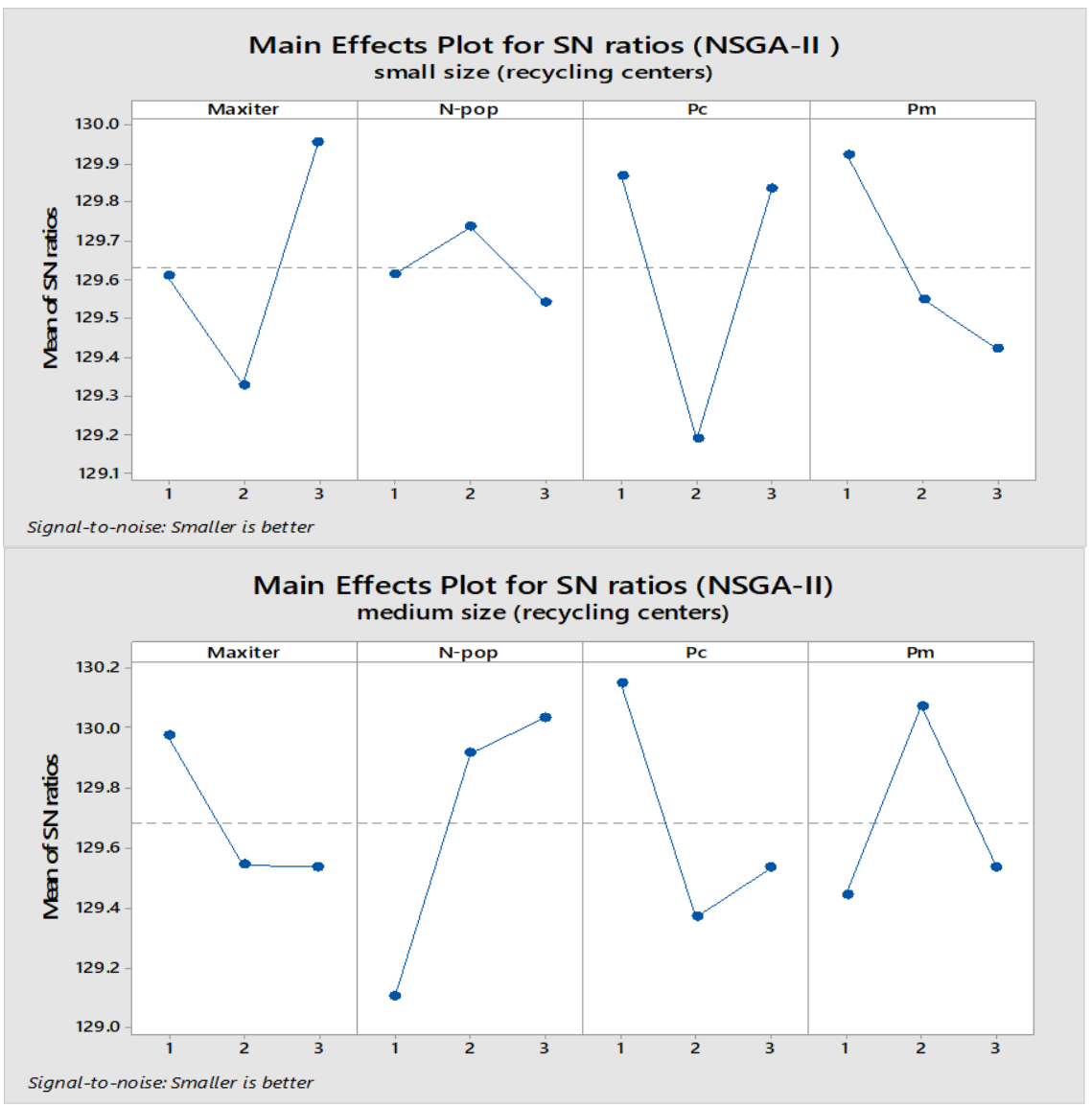




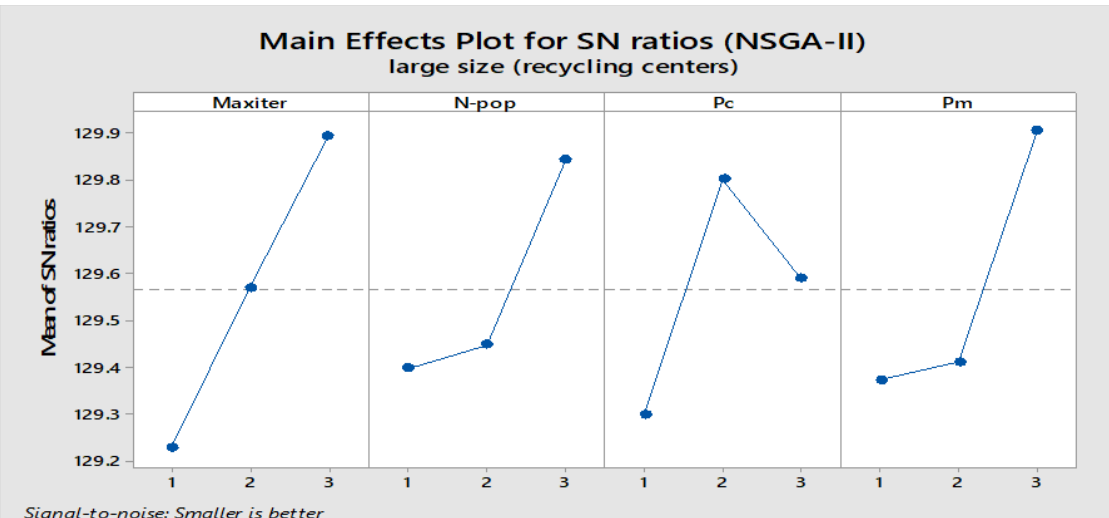

Signal-to-noise: Smaller is better

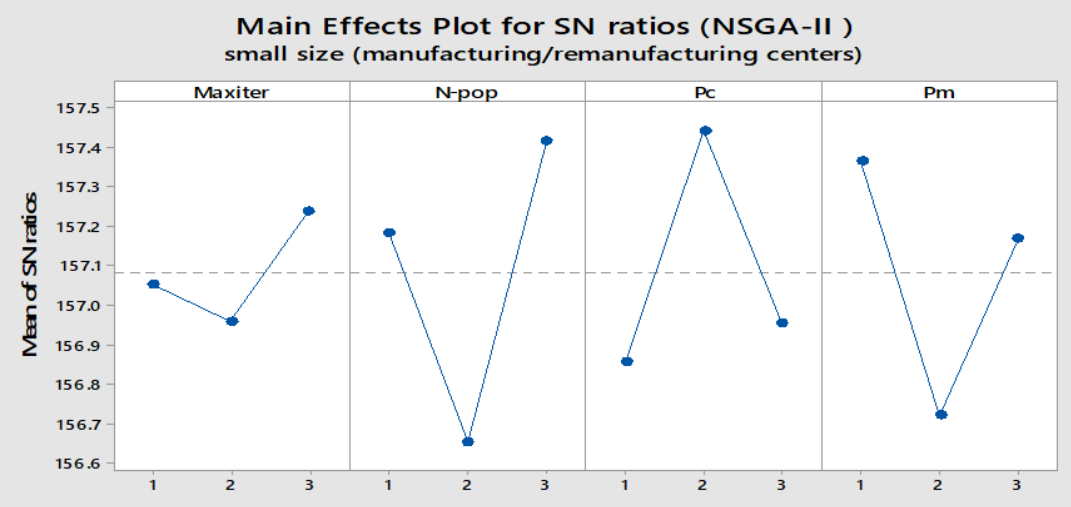

Signal-to-noise: Smaller is better

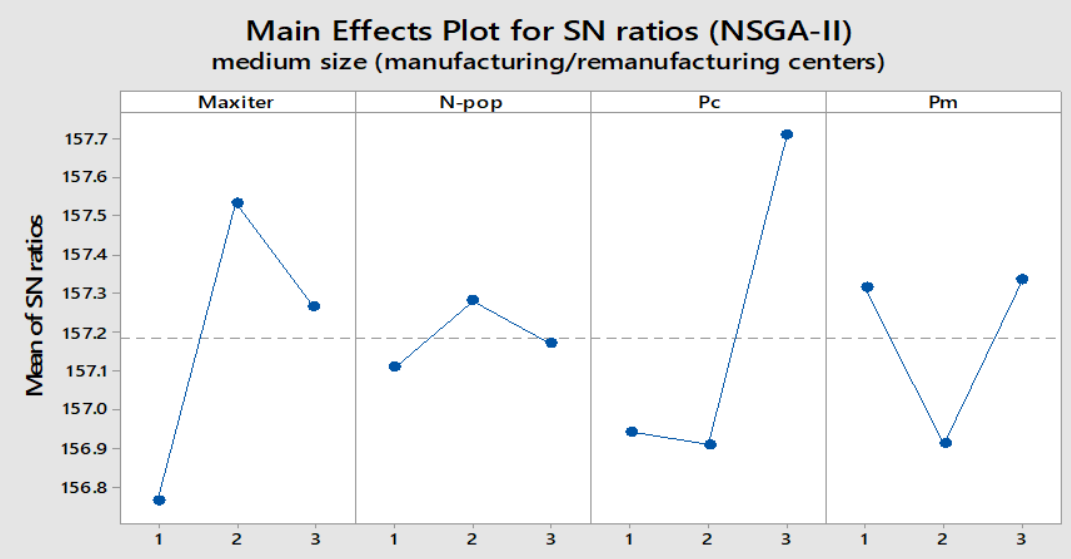

Signal-to-noise: Smaller is better

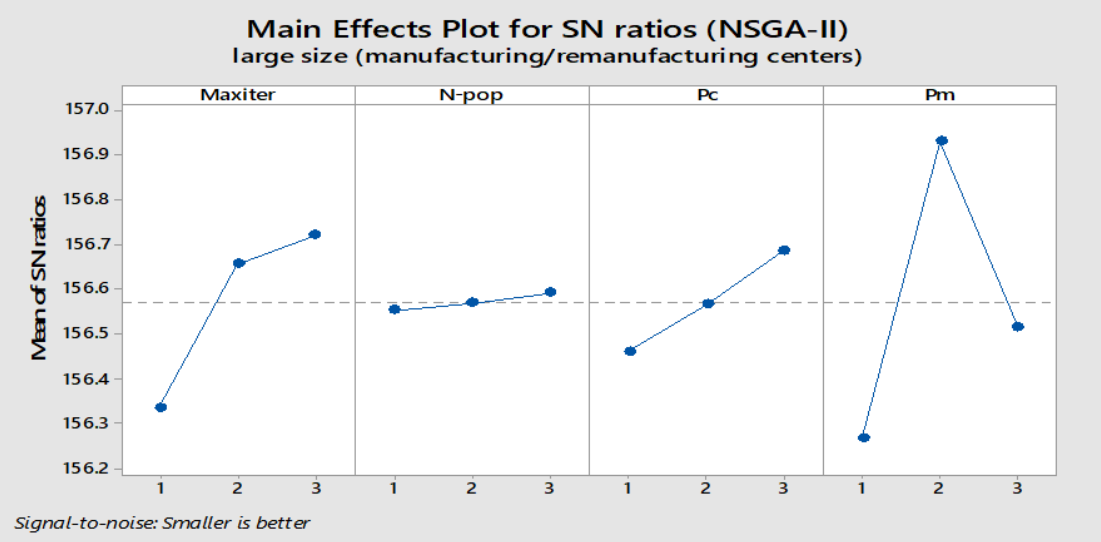



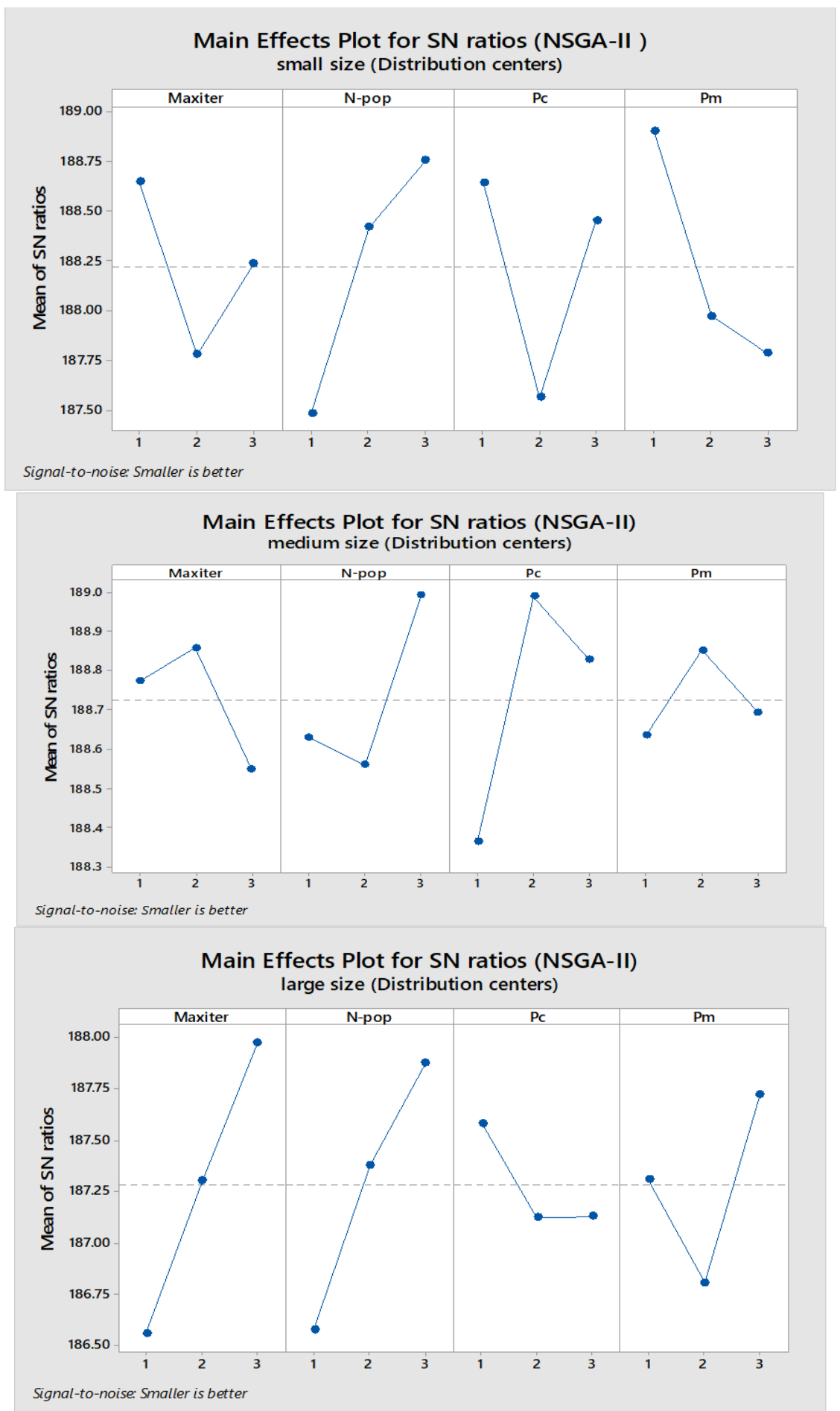

Figure 5: The $\mathrm{S} / \mathrm{N}$ ratios

Table 9: The best parameters values of the NSGA-II algorithm

\begin{tabular}{|c|c|c|c|c|c|c|c|c|c|c|}
\hline \multirow{2}{*}{ NSGA-II } & \multirow{2}{*}{ Parameters } & \multicolumn{3}{|c|}{ Small } & \multicolumn{3}{|c|}{ Medium } & \multicolumn{3}{|c|}{ Large } \\
\hline & & Level 1 & Level 2 & Level 3 & Level 1 & Level 2 & Level 3 & Level 1 & Level 2 & Level 3 \\
\hline
\end{tabular}




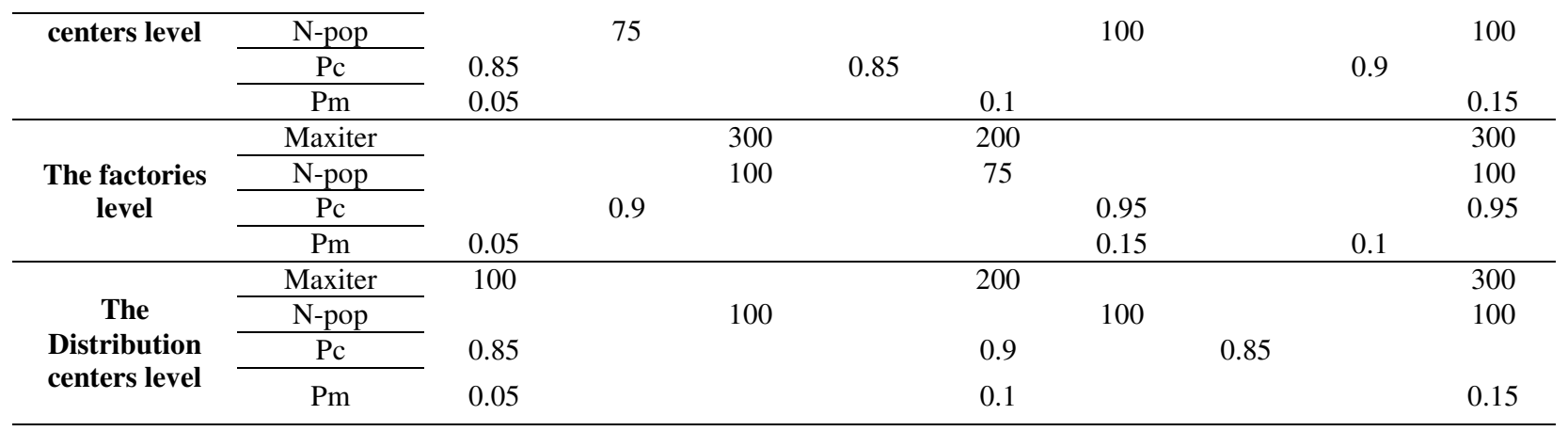

\section{5-3- The weight coefficients in LP-metric method}

It is necessary to determine the suitable weight coefficients of the objective functions for executing the proposed model in GAMS software based on the LP-metric method. Different weight combinations are solved for a small-size determinant problem, and the appropriate weight is generalized to the other problem. Figure 6 illustrates how the value of the objective functions is affected by various weights in different levels of the problem. The most significant variations in the objective functions happen in the weight coefficients between 0.4 and 0.9 , and in the other weights, the objective function value becomes zero. So, it is better to weigh the goals between these limits. According to Figure 6 , a weight of 0.8 is chosen for the first objective function $\left(F_{1}^{R}\right.$ ) and a weight of 0.2 for the second objective function $\left(F_{2}^{R}\right)$ of the FLM. A weight of 0.7 is chosen for the first objective function $\left(F_{1}{ }^{F}\right)$ and a weight of 0.3 for the second objective function $\left(F_{2}^{F}\right)$ of the SLM. Moreover, a weight of 0.9 is chosen for the first objective function $\left(F_{1}^{D}\right)$ and a weight of 0.1 for the second objective function $\left(F_{2}^{D}\right)$ of the TLM.
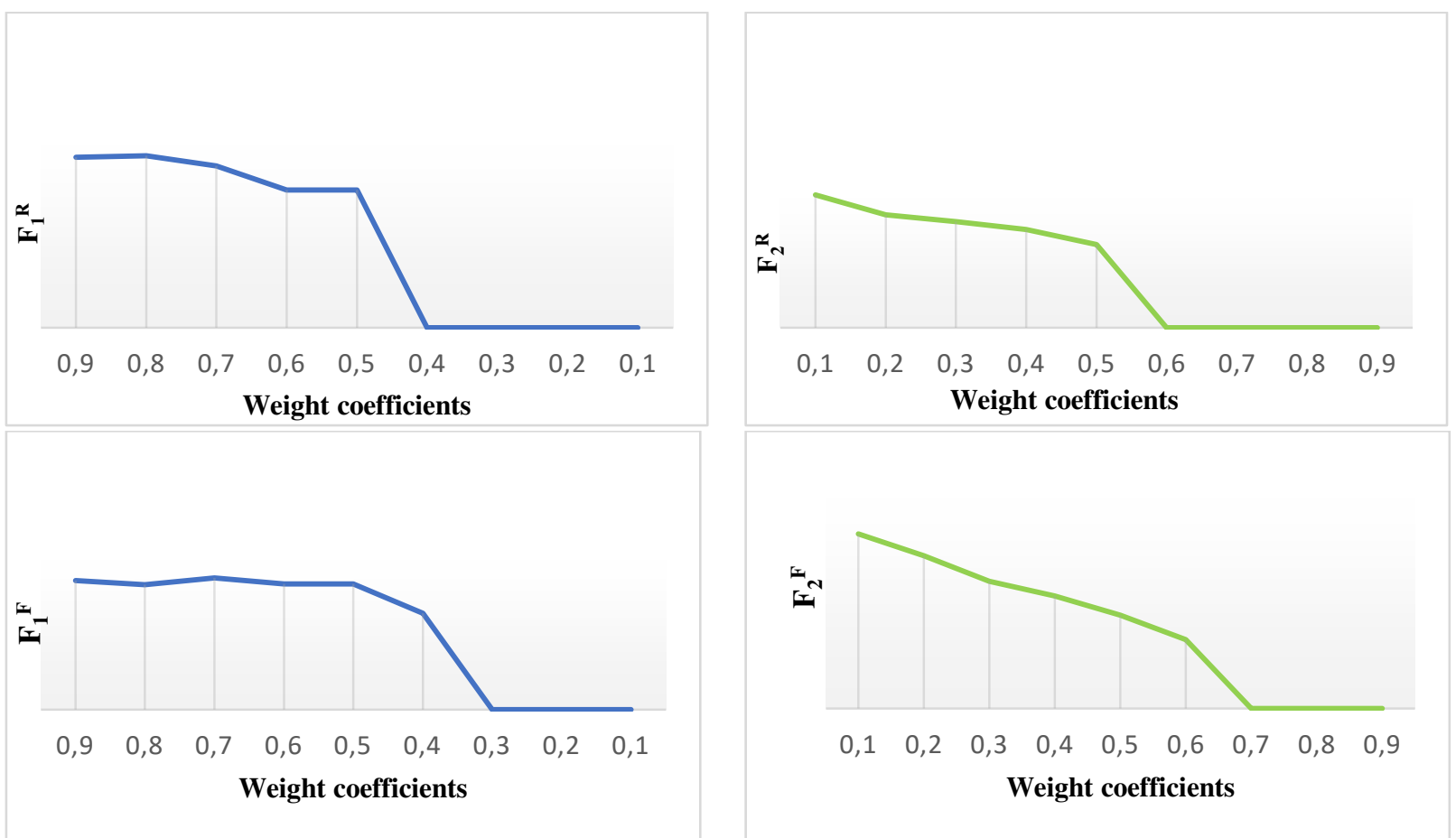

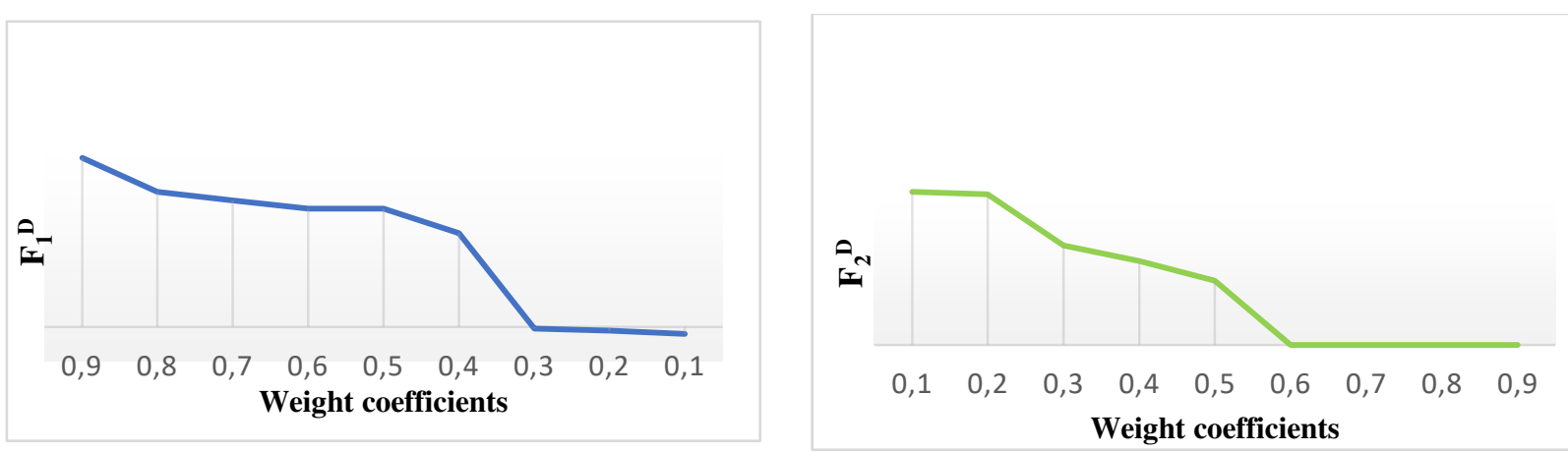

Figure 6: The different weights in the various levels of the problem

\section{5-4- Validation of the NSGA-II algorithm}

The LP-metric method is used for model validation. Thus, six small-scale problems are solved in GAMS software with the LP-metric method. Note that it is not possible for large-scale problems. Moreover, for comparison, the six small problems are solved with the NSGA-II algorithm. Since the NSGA-II is run based on the assumption that the initial population is randomly generated; hence, any test problem is solved ten times. The best non-dominated solution is selected from the ten times of running results, and its LP-metric measure is calculated. The measured value is compared with the value obtained from the exact method that is run in GAMS. In Table 10, the relative gap between the values of the two LP-metric measures, which are achieved from the exact method and the NSGA-II algorithm, is calculated.

Table 10: Validation results

\begin{tabular}{|c|c|c|c|c|c|c|c|}
\hline \multirow{2}{*}{ 要 } & \multirow{2}{*}{$\begin{array}{c}\text { Dimension of the } \\
\text { problems }\end{array}$} & \multirow{2}{*}{$-\frac{5}{2}$} & \multicolumn{2}{|c|}{ GAMS } & \multicolumn{3}{|c|}{ NSGA-II } \\
\hline & & & LP- Metric & $\begin{array}{l}\text { CPU Time } \\
\text { (S) }\end{array}$ & LP- Metric & $\begin{array}{c}\text { CPU Time } \\
\text { (S) }\end{array}$ & Relative GAP \\
\hline \multirow{3}{*}{1} & \multirow{3}{*}{$k_{2} i_{2} j_{2} p_{2} c_{2} v_{2} t_{2}$} & 1 & 0.275 & \multirow{3}{*}{5} & 0.301 & \multirow{3}{*}{73} & $9 \%$ \\
\hline & & 2 & 0.425 & & 0.453 & & $6 \%$ \\
\hline & & 3 & 0.267 & & 0.272 & & $1 \%$ \\
\hline \multirow{3}{*}{2} & \multirow{3}{*}{$k_{3} i_{3} j_{5} p_{3} c_{3} v_{3} t_{2}$} & 1 & 0.028 & \multirow{3}{*}{13} & 0.0299 & \multirow{3}{*}{98} & $6 \%$ \\
\hline & & 2 & 0.775 & & 0.834 & & $7 \%$ \\
\hline & & 3 & 0.093 & & 0.094 & & $1 \%$ \\
\hline \multirow{3}{*}{3} & \multirow{3}{*}{$k_{3} i_{4} j_{5} p_{3} c_{3} v_{3} t_{4}$} & 1 & 0.379 & \multirow{3}{*}{66} & 0.411 & \multirow{3}{*}{134} & $8 \%$ \\
\hline & & 2 & 0.647 & & 0.691 & & $6 \%$ \\
\hline & & 3 & 0.093 & & 0.095 & & $2 \%$ \\
\hline \multirow{3}{*}{4} & \multirow{3}{*}{$k_{4} i_{4} j_{5} p_{3} c_{3} v_{3} t_{5}$} & 1 & 0.446 & \multirow{3}{*}{386} & 0.481 & \multirow{3}{*}{176} & $7 \%$ \\
\hline & & 2 & 0.626 & & 0.679 & & $8 \%$ \\
\hline & & 3 & 0.290 & & 0.301 & & $3 \%$ \\
\hline \multirow{3}{*}{5} & \multirow{3}{*}{$k_{4} i_{4} j_{5} p_{3} c_{4} v_{3} t_{6}$} & 1 & 0.599 & \multirow{3}{*}{410} & 0.657 & \multirow{3}{*}{241} & $9 \%$ \\
\hline & & 2 & 0.700 & & 0.769 & & $9 \%$ \\
\hline & & 3 & 0.145 & & 0.149 & & $2 \%$ \\
\hline \multirow{3}{*}{6} & \multirow{3}{*}{$k_{4} i_{4} j_{5} p_{3} c_{6} v_{4} t_{7}$} & 1 & 0.553 & \multirow{3}{*}{924} & 0.602 & \multirow{3}{*}{358} & $8 \%$ \\
\hline & & 2 & 0.813 & & 0.869 & & $6 \%$ \\
\hline & & 3 & 0.209 & & 0.216 & & $3 \%$ \\
\hline
\end{tabular}

According to Table 10, the average of the relative gaps is $7 \%, 7 \%$, and $1 \%$ for the three levels, respectively. Therefore, we can declare that the NSGA-II algorithm is suitable to solve the considered problem. 


\section{5-5- Analysis of MC Simulation}

In this research, it is assumed that the demand for remanufactured and new products in distribution centers at different periods are stochastic. The probability distribution function associated with the value of demand is uniform. The MC simulation approach is used to deal with the uncertainty

Equation (53) is used to calculate the number of simulation replications. First, a pilot run of MC simulation is implemented considering an initial sample size of 10 replications to calculate mean and the standard deviation of $F_{1}^{D}$ and $F_{2}^{D}$ for a chromosome. By considering a confidence level of $95 \%$ and an error of 0.1 , the number of simulation replications is obtained equal to eight based on equation (53).

The medium and large-size problems are solved to compare the NSGA-II and MCNSGA-II algorithms. Figure 7 and Figure 8 indicate the non-dominated solutions, which are achieved from the NSGA-II and MCNSGA-II algorithms for the medium and large-size problems. The stochastic demand is the parameter of the third level of the considered SC; thus, the nondominated solutions of the third level are only shown in Figure 7 and Figure 8.

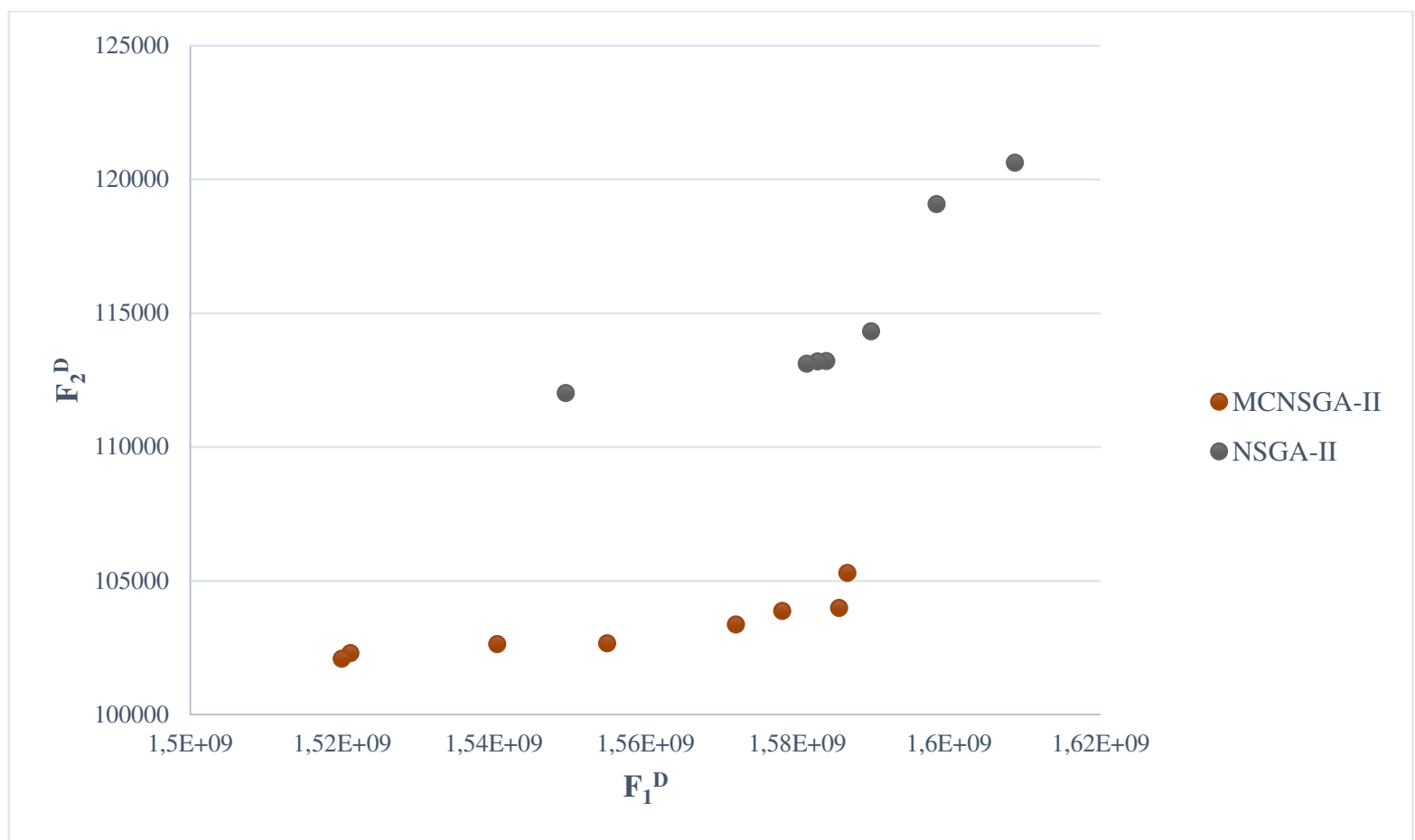

Figure 7: Comparison between the non-dominated solutions obtained with different optimization methods for a medium-size instance 


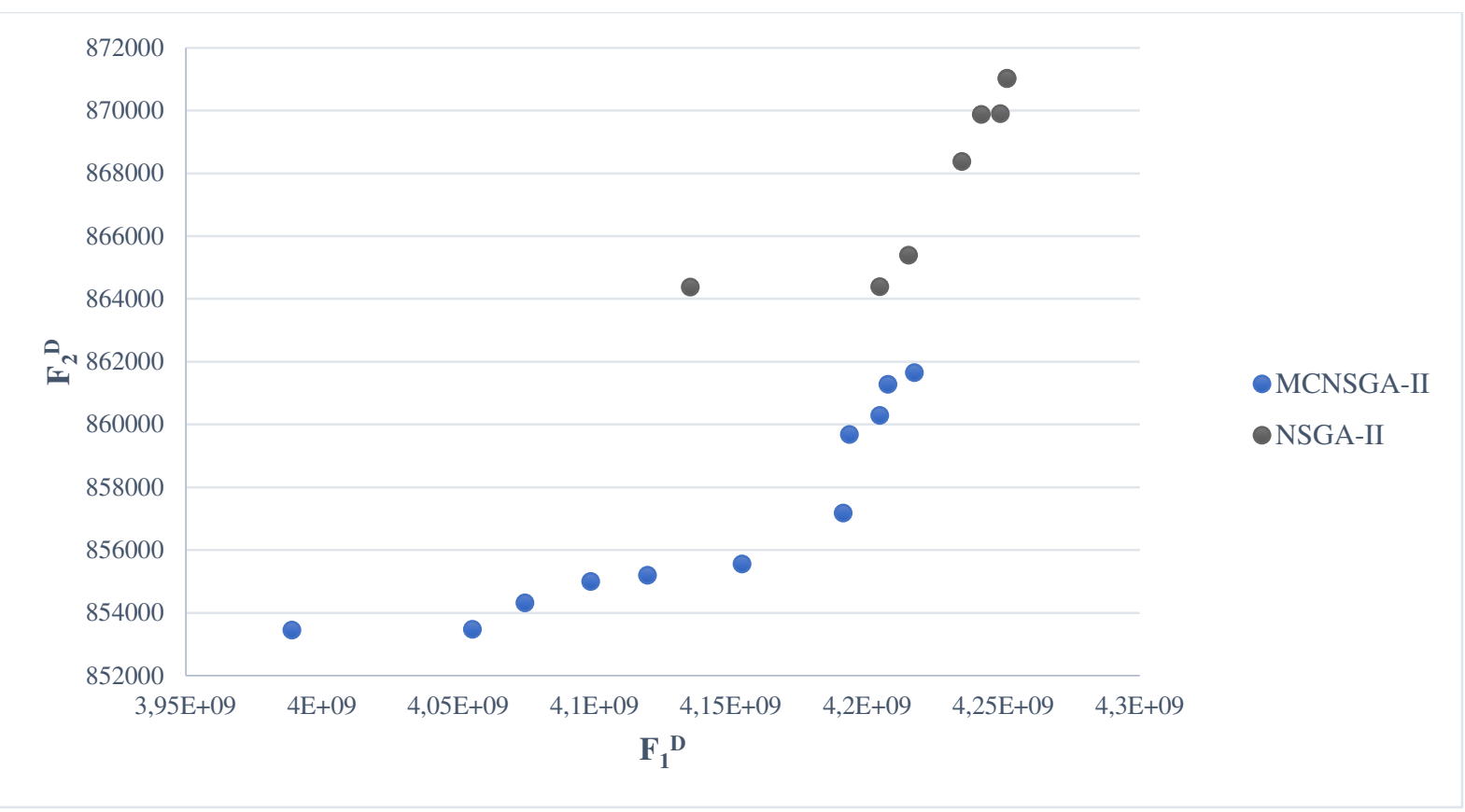

Figure 8: Comparison between the non-dominated solutions obtained with different optimization methods for a large-size instance

Table 11 and Table 12 indicates the value of convergence (C) and spacing (S) metrics of the different optimization methods. As can be seen, the MCNSGA-II algorithm presents better non-dominated solutions than the NSGA-II algorithm.

Table 11:Convergence metric (C)

\begin{tabular}{|c|c|c|c|c|}
\hline \multirow{2}{*}{$C(A, B)$} & \multicolumn{2}{|c|}{ Medium-size instance } & \multicolumn{2}{|c|}{ Large-size instance } \\
\hline & NSGA-II & MCNSGA-II & NSGA-II & MCNSGA-II \\
\hline NSGA-II & - & 0 & - & 0 \\
\hline MCNSGA-II & 0.5714 & - & 0.375 & - \\
\hline
\end{tabular}

Table 12: Spacing metric (S)

\begin{tabular}{cccccc}
\hline \multirow{2}{*}{$S$} & \multicolumn{2}{c}{ Medium-size instance } & & \multicolumn{2}{c}{ Large-size instance } \\
\cline { 2 - 3 } \cline { 5 - 6 } & NSGA-II & MCNSGA-II & & NSGA-II & MCNSGA-II \\
\cline { 2 - 3 } & $1.01 \mathrm{E}+07$ & $5.45 \mathrm{E}+06$ & & $2.16 \mathrm{E}+07$ & $1.82 \mathrm{E}+07$ \\
\hline
\end{tabular}

\section{6- Conclusions}

In this paper, we considered an integrated PD planning problem of a multi-level GCLSC system, that includes multiple recycling centers, multiple remanufacturing/manufacturing centers, and multiple distribution centers. A three-level bi-objective MIP model is presented to maximize profit and minimize the amount of greenhouse gas emissions. A hierarchical iterative approach using the LP-metric method and the NSGA-II algorithm is suggested to solve the proposed model. The Taguchi experimental design approach is applied to find optimum control parameters of NSGA-II. Moreover, the NSGA-II algorithm is fusioned with MC simulation 
(MCNSGA-II) to deal with the uncertainty of the product demand in distribution centers. The results obtained show that the simulation-optimization approach presented better results than the deterministic approach. Furthermore, there are several opportunities in future research. First, to understand the realities of the environment, it is important to consider uncertainty in other influential parameters such as purchase costs, shipping costs, and so on. Second, studying the literature on multi-level production-distribution planning shows that fewer study goals, such as customer satisfaction and reduction of delay time in the model, were considered. Adding the aforementioned goals could be of interest for future research. Third, in this and other studies, multi-level planning has been solved at two or three levels. Adding the fourth level as the supplier level can be an appropriate area for future research.

\section{Compliance with ethical standards}

Conflict of interest The authors declare that they have no conflict of interest regarding the publication of this paper.

Ethical approval This article does not contain any studies with human participants or animals performed by any of the authors. 


\section{Appendix}

Table 13: Definition of the sets

\begin{tabular}{ll}
\hline$T$ & Set of periods $(t=1,2, \ldots, T)$ \\
$P$ & Set of product types $(p=1,2, \ldots, P)$ \\
$C$ & Set of component types $(c=1,2, \ldots, C)$ \\
$V$ & Set of vehicle types $(v=1,2, \ldots, V)$ \\
$I$ & Set of manufacturing/remanufacturing factory $(i=1,2, \ldots, I)$ \\
$J$ & Set of distributors $(j=1,2, \ldots, J)$ \\
$K$ & Set of recycling centers $(k=1,2, \ldots, K)$ \\
\hline
\end{tabular}

Table 14: The parameters of the FLM

\begin{tabular}{|c|c|}
\hline$P P C_{k i c t}$ & Purchase unit cost at period $t$ for eligible component $c$ by factory $i$ from recycling center $k$ \\
\hline$U R C C_{j k p t}$ & Recycling unit cost at period $t$ for returned product $p$ from distributor $j$ by recycling center $k$ \\
\hline$S D T_{k p t}$ & Set-up cost at period $t$ for returned product $p$ in recycling center $k$ \\
\hline$U D T C_{k p t}$ & Disassembly and tested unit cost of at period $t$ for returned product $p$ in recycling center $k$ \\
\hline$U D C_{k p t}$ & Disposing unit cost at period $t$ for component $c$ in recycling center $k$ \\
\hline$I C Q C_{k c t}^{R}$ & Inventory unit cost at period $t$ for eligible component $c$ in recycling center $k$ \\
\hline$I C R P_{k p t}^{R}$ & Inventory unit cost at period $t$ for returned product $p$ in recycling center $k$ \\
\hline$U T C_{k i c v t}^{R F}$ & $\begin{array}{l}\text { Transportation unit cost at period } t \text { for eligible component } c \text { by vehicle } v \text { from recycling center } k \text { to factory } \\
i\end{array}$ \\
\hline$B O C_{p c}$ & Bill of component $c$ to product $p$ \\
\hline$\theta_{k c t}$ & Rate of remanufacture for component $c$ at period $t$ in recycling center $k$ \\
\hline $\bar{\alpha}_{k p}^{R}$ & The highest inventory quantity of returned product $p$ in recycling center $k$ \\
\hline $\bar{\beta}_{k c}^{R}$ & The highest inventory quantity of eligible components $c$ in recycling center $k$ \\
\hline$M D T_{k p}^{R}$ & $\begin{array}{l}\text { The highest amount of returned product } p \text { can be disassembled and tested in the recycling center } k \text { in every } \\
\text { period }\end{array}$ \\
\hline $\operatorname{cap}_{v}^{R F}$ & Capacity of vehicle $v$ that goes from recycling centers to manufacturing/remanufacturing factories \\
\hline $\operatorname{dis}_{k i}^{R F}$ & Distance between recycling center $k$ and factory $i$ \\
\hline Emis $_{k i c v t}^{R F}$ & $\begin{array}{l}\text { Greenhouse gas emissions per unit of eligible component } c \text { with vehicle } v \text { per kilometer between recycling } \\
\text { center } k \text { and factory } i\end{array}$ \\
\hline$T E_{t}^{\max R F}$ & $\begin{array}{l}\text { Maximum greenhouse gas emissions allowance for transportation system between recycling centers and } \\
\text { factories at period } t\end{array}$ \\
\hline
\end{tabular}

Table 15: The parameters of the SLM

$M P N_{i j p t} \quad$ Average price at period $t$ for new product $p$ paid by distributor $j$ to factory $i$


$M P R_{i j p t}$

$S A_{i p t}$

$S R A_{i p t}$

$U A C_{i p t}$

$U R A C_{i p t}$

$S P_{i c t}$

$S R P_{i c t}$

$U P C_{i c t}$

$U R P C_{i c t}$

${ }_{I C N P} P_{i p t}^{F}$

ICRMP $_{i p t}^{F}$

${ }_{I C Q C}^{F}{ }_{i c t}^{F}$

$I C N C_{i c t}^{F}$

$\operatorname{ICR} C_{i c t}^{F}$

$U T C_{i j p v t}^{F D}$

$\bar{\beta}_{i c}^{F}$

$\bar{\zeta}_{i c}^{F}$

$\bar{\xi}_{i c}^{F}$

$\bar{\lambda}_{i p}^{F}$

$-F$

$x_{\text {ip }}$

$M A_{i p}$

$M R A_{i p}$

$M P_{i c}$

$M R P_{i c}$

$\operatorname{cap}_{v}^{F D}$

$d i s_{i j}^{F D}$

Emis $_{i j p v t}^{F D}$

Emispnc $_{i c t}^{F}$

Emisprc $_{i c t}^{F}$

$O P E_{t}^{\max F}$
Average price at period $t$ for remanufactured product $p$ paid by distributor $j$ to factory $i$

Set-up cost at period $t$ to assemble new product $p$ in factory $i$

Set-up cost at period $t$ to assemble remanufactured product $p$ in factory $i$

Assembly unit cost at period $t$ for new product $p$ in factory $i$

Assembly unit cost at period $t$ for remanufactured product $p$ in factory $i$

Set-up cost at period $t$ when component $c$ is processed in factory $i$

Set-up cost at period $t$ when component $c$ is reprocessed in factory $i$

Processing unit cost at period $t$ for component $c$ in factory $i$

Reprocessing unit cost at period $t$ for component $c$ in factory $i$

Inventory unit cost at period $t$ for new product $p$ in factory $i$

Inventory unit cost at period $t$ for remanufactured product $p$ in factory $i$

Inventory unit cost at period $t$ for eligible component $c$ in factory $i$

Inventory unit cost at period $t$ for new component $c$ in factory $i$

Inventory unit cost at period $t$ for remanufactured component $c$ in factory $i$

Transportation unit cost at period $t$ for product $p$ by vehicle $v$ from factory $i$ to distributor $j$

The highest inventory quantity of eligible components $c$ in factory $i$

The highest inventory quantity of new components $c$ in factory $i$

The highest inventory quantity of remanufactured components $c$ in factory $i$

The highest inventory quantity of new products $p$ in factory $i$

The highest inventory quantity of remanufactured products $p$ in factory $i$

Maximum capacity of factory $i$ to assemble new product $p$ at each period

Maximum capacity of factory $i$ to assemble remanufactured product $p$ at each period

Maximum capacity of factory $i$ to process component $c$ at each period

Maximum capacity of factory $i$ to reprocess component $c$ at each period

Capacity of vehicle $v$ that goes from a manufacturing/ remanufacturing factory to a distributor

Distance between factory $i$ and distributor $j$

Greenhouse gas emissions per unit of product $p$ with vehicle $v$ per kilometer between factory $i$ and distributor $j$ at period $t$

Greenhouse gas emissions during processing of each new component $c$ in manufacturing/ remanufacturing factory $i$ at period $t$

Greenhouse gas emissions during the reprocessing of each component $c$ in manufacturing/ remanufacturing factory $i$ at period $t$

Maximum greenhouse gas emissions allowance at period $t$ during processing/reprocessing operations in factories 


$\begin{array}{ll}T E_{t}^{\max F D} & \begin{array}{l}\text { Maximum greenhouse gas emissions allowance for transportation system between factories and } \\ \text { distribution centers at period } t\end{array} \\ P P C_{k i c t} & \text { This notation has taken value in the FLM } \\ B O C_{p c} & \text { This notation has taken value in the FLM }\end{array}$

Table 16: The parameters of the TLM

\begin{tabular}{|c|c|}
\hline$S P N_{j p t}$ & Selling price at period $t$ for new product $p$ in distributor $j$ \\
\hline$S P R_{j p t}$ & Selling price at period $t$ for remanufactured product $p$ in distributor $j$ \\
\hline$D N M_{j p t}$ & Demands of new product $p$ at period $t$ in the market of distributor $j$ \\
\hline$D R M_{j p t}$ & Demands of remanufactured product $p$ at period $t$ in the market of distributor $j$ \\
\hline$U R C D_{j p t}$ & Recycling unit cost at period $t$ for returned product $p$ paid by distributor $j$ to retailers or customers \\
\hline$U S N P_{j p t}$ & Shortage unit cost at period $t$ for new product $p$ paid by distributor $j$ \\
\hline$U S R P_{j p t}$ & Shortage unit cost at period $t$ for remanufactured product $p$ paid by distributor $j$ \\
\hline$I C N P_{j p t}^{D}$ & Inventory unit cost at period $t$ for new product $p$ in distributor $j$ \\
\hline $\operatorname{ICR} M_{j p t}^{D}$ & Inventory unit cost at period $t$ for remanufactured product $p$ in distributor $j$ \\
\hline$I C R P_{j p t}^{D}$ & Inventory unit cost at period $t$ for returned product $p$ in distributor $j$ \\
\hline$U T C_{j k p v t}^{D R}$ & $\begin{array}{l}\text { Transportation unit cost at period } t \text { for returned product } p \text { by vehicle } v \text { from distributor } j \text { to recycling } \\
\text { center } k\end{array}$ \\
\hline$E P A_{j p t}$ & Amount of returned product $p$ at period $t$ available in the market of distributor $j$ \\
\hline $\bar{\lambda}_{j p}^{D}$ & The highest inventory quantity of new product $p$ in distributor $j$ \\
\hline $\bar{x}_{j p}^{D}$ & The highest inventory quantity of remanufactured product $p$ in distributor $j$ \\
\hline$-\alpha_{j p}$ & The highest inventory quantity of return product $p$ in distributor $j$ \\
\hline $\operatorname{cap}_{v}^{D R}$ & Capacity of vehicle $v$ that goes from distribution to recycling centers \\
\hline $\operatorname{dis}_{j k}^{D R}$ & Distance between distributor $j$ and recycling center $k$ \\
\hline Emis ${ }_{j k p v t}^{D R}$ & $\begin{array}{l}\text { Greenhouse gas emissions per unit of product } p \text { with vehicle } v \text { per kilometer between distributor } j \text { and } \\
\text { recycling center } k \text { at period } t\end{array}$ \\
\hline$T E_{t}^{\max D R}$ & $\begin{array}{l}\text { Maximum greenhouse gas emissions allowance for transportation system between distribution and } \\
\text { recycling centers at period } t\end{array}$ \\
\hline$U R C C_{j k p t}$ & This notation has taken value in the FLM \\
\hline$M_{P N} N_{i j p t}$ & This notation has taken value in the SLM \\
\hline$M P R_{i j p t}$ & This notation has taken value in the SLM \\
\hline
\end{tabular}

Table 17: The variables of the FLM

$a f_{k i c v t}$

Amount of eligible component $c$ that is transported by vehicle $v$ at period $t$ from recycling center $k$ to 


\begin{tabular}{ll}
\hline$d a_{j k p v t}$ & $\begin{array}{l}\text { factory } i \\
\text { Amount of returned product } p \text { that is transported by vehicle } v \text { at period } t \text { from distributor } j \text { to recycling } \\
\text { center } k\end{array}$ \\
$d_{k p t}$ & Amount of returned product $p$ at period $t$ that is disassembled and tested in recycling center $k$ \\
$\alpha_{k p t}^{R}$ & Amount of component $c$ at period $t$ that is disposed of in recycling center $k$ \\
$\beta_{k c t}^{R}$ & Inventory quantity of returned product $p$ at recycling center $k$ at the end of period $t$ \\
$\sigma_{k p t}$ & Inventory quantity of eligible component $c$ in recycling center $k$ at the end of period $t$ \\
\hline
\end{tabular}

Table 18: The variables of the SLM

\begin{tabular}{|c|c|}
\hline$f d n_{i j p v t}$ & Amount of new product $p$ at period $t$ that is transported by vehicle $v$ from factory $i$ to distributor $j$ \\
\hline$f d r_{i j p v t}$ & $\begin{array}{l}\text { Amount of remanufactured product } p \text { at period } t \text { that is transported by vehicle } v \text { from factory } i \text { to } \\
\text { distributor } j\end{array}$ \\
\hline$x_{i p t}$ & Amount of new product $p$ at period $t$ that is assembled in factory $i$ \\
\hline$y_{i p t}$ & Amount of remanufactured product $p$ at period $t$ that is assembled in factory $i$ \\
\hline$w_{i c t}$ & Amount of component $c$ at period $t$ that is processed in factory $i$ \\
\hline$z_{i c t}$ & Amount of component $c$ at period $t$ that is reprocessed in factory $i$ \\
\hline$\lambda_{i p t}^{F}$ & Inventory quantity of new product $p$ at factory $i$ at the end of period $t$ \\
\hline$x_{i p t}^{F}$ & Inventory quantity of remanufactured product $p$ in factory $i$ at the end of period $t$ \\
\hline$\beta_{i c t}^{F}$ & Inventory quantity of eligible component $c$ in factory $i$ at the end of period $t$ \\
\hline$\zeta_{i c t}^{F}$ & Inventory quantity of new component $c$ in factory $i$ at the end of period $t$ \\
\hline$\xi_{i c t}^{F}$ & Inventory quantity of remanufactured component $c$ in factory $i$ at the end of period $t$ \\
\hline$\eta_{i p t}$ & 1 if new product $p$ is assembled by factory $i$ at period $t ; 0$ otherwise \\
\hline$\delta_{i p t}$ & 1 if remanufactured product $p$ is assembled by factory $i$ in batches at period $t ; 0$ otherwise \\
\hline$\pi_{i c t}$ & 1 if component $c$ is processed by factory $i$ at period $t ; 0$ otherwise \\
\hline$\tau_{i c t}$ & 1 if component $c$ is reprocessed by factory $i$ at period $t ; 0$ otherwise \\
\hline subc $_{i c t}$ & The amount of one-way substitution for component $c$ at period $t$ in factory $i$ \\
\hline$a f_{k i c v t}$ & This notation has taken value in the FLM \\
\hline
\end{tabular}

Table 19: The variables of the TLM

\begin{tabular}{ll}
\hline$n S S_{j p t}$ & Amount of new shortage product $p$ at period $t$ in distributor $j$ \\
$r S S_{j p t}$ & Amount of remanufactured shortage product $p$ at period $t$ in distributor $j$ \\
$\gamma_{j p t}$ & Amount of returned product $p$ at period $t$ that is collected by distributor $j$ from downstream markets \\
$\lambda_{j p t}^{D}$ & Inventory quantity of new product $p$ in distributor $j$ at the end of period $t$
\end{tabular}


$x \underset{j p t}{D}$

$\alpha_{j p t}^{D}$

$d a_{j k p v t}$

$f d n_{i j p v t}$

$f d r_{i j p v t}$
Inventory quantity of remanufactured product $p$ in distributor $j$ at the end of period $t$

Inventory quantity of return product $p$ in distributor $j$ at the end of period $t$

This notation has taken value in the FLM

This notation has taken value in the SLM

This notation has taken value in the SLM

\section{Compliance with ethical standards}

Conflict of interest The authors declare that they have no conflict ofinterest.

\section{References}

Barbarosoğlu, G., \& Özgür, D. (1999). Hierarchical design of an integrated production and 2-echelon distribution system. European Journal of Operational Research, 118(3), 464-484.

Brandimarte, P. (2014). Handbook in Monte Carlo simulation: applications in financial engineering, risk management, and economics: John Wiley \& Sons.

Casas-Ramírez, M. S., Camacho-Vallejo, J. F., González-Ramírez, R. G., Marmolejo-Saucedo, J. A., \& Velarde-Cantú, J. M. (2018). Optimizing a Biobjective Production-Distribution Planning Problem Using a GRASP. Complexity.

Chen, M., \& Wang, W. (1997). A linear programming model for integrated steel production and distribution planning,. International Journal of Operations \& Production Management, 17(6), 592-610

Deb, K., Pratap, A., Agarwal, S., \& Meyarivan, T. (2002). A fast and elitist multiobjective genetic algorithm: NSGA-II. IEEE transactions on evolutionary computation, 6(2), 182-197.

Dekker, R., Bloemhof, J., \& Mallidis, I. (2012). Operations Research for green logisticseAn overview of aspects, issues, contributions and challenges. European Journal of Operational Research, 219(3), 671-679.

Devapriya, P., Ferrell, W., \& Geismar, N. (2017). Integrated Production and Distribution Scheduling with a Perishable Product. European Journal of Operational Research, 259(3), 906-916.

Ensafian, H., \& Yaghoubi, S. (2017). Robust optimization model for integrated procurement, production and distribution in platelet supply chain. Transportation Research Part E: Logistics and Transportation Review, 103, 32-55.

Fahimnia, B., Farahani, R. Z., Marian, R., \& Luong, L. (2013). A review and critique on integrated production-distribution planning models and techniques. Journal of Manufacturing Systems, 32, $1-19$.

Farahani, M., \& Rahmani, D. (2017). Production and distribution planning in petroleum supply chains regarding the impacts of gas injection and swap. Energy, 141, 991-1003.

Geoffrion, A. M., \& Graves, G. W. (1974). Multicommodity distribution system design by Benders decomposition. Management Science, 20(5), 822-844.

Goodarzian, F., Shishebori, D., Nasseri, H., \& Dadvar, F. (2021). A bi-objective production-distribution problem in a supply chain network under grey flexible conditions. RAIRO-Operations Research, 55, 1287-1316.

Hansen, P., Jaumard, B., \& Savard, G. (1992). New branch and bound rules for linear bilevel programming. SIAM Journal on Science and Statistical Computing, 13(5), 1194-1217. 
Haq, A. N., Vrat, P., \& Kanda, A. (1991). An integrated production-inventory-distribution model for manufacture of urea: a case. International Journal of Production Economics, 25, 39-49.

Jing, Y., \& Li, W. (2018). Integrated recycling-integrated production-distribution planning for decentralized closed-loop supply chain. Journal of Industrial \& Management Optimization, 14(2), 511-539.

Kang, H. Y., Pearn, W. L., Chung, I. P., \& Lee, A. H. (2016). An enhanced model for the integrated production and transportation problem in a multiple vehicles environment. Soft Computing, 20(4), $1415-1435$.

Lee, Y. H., \& Kim, S. H. Optimal production-distribution planning in supply chain management using a hybrid simulation-analytic approach. In Simulation Conference, 2000 (Vol. 2, pp. 1252-1259): IEEE

Liang, T. F., \& Cheng, H. W. (2009). Application of fuzzy sets to manufacturing/distribution planning decisions with multi-product and multi-time period in supply chains. Expert Systems with Application,, 36(2), 3367-3377.

Lin, C., Choy, K. L., \& Chung, S. H. (2014). Survey of green vehicle routing problem: past and future trends. Expert Systems with Applications, 41(1), 1118-1138.

Ma, Y., Yan, F., Kang, K., \& Wei, X. (2016). A novel integrated production-distribution planning model with conflict and coordination in a supply chain network. Knowledge-Based Systems, 105, 119133.

Moon, I., Jeong, Y. J., \& Saha, S. (2016). Fuzzy Bi-Objective Production-Distribution Planning Problem under the Carbon Emission Constraint. sustainability, 8(8), 798.

Nasiri, G. R., Zolfaghari, R., \& Davoudpour, H. (2014). An integrated supply chain productiondistribution planning with stochastic demands. Computers \& Industrial Engineering, 77, 35-45.

Niknamfar, A. H., Niaki, S. T. A., \& Pasandideh, S. H. R. (2015). Robust optimization approach for an aggregate production- distribution planning in a three-level supply chain. The International Journal of Advanced Manufacturing Technology, 76(1-4), 623-634

Nourifar, R., Mahdavi, I., Mahdavi-Amiri, N., \& Paydar, M. M. (2018). Optimizing decentralized production-distribution planning problem in a multi-period supply chain network under uncertainty. Journal of Industrial Engineering International, 14(2), 367-382.

Osorio, A. F., Brailsford, S. C., Smith, H. K., Forero-Matiz, S. P., \& Camacho-Rodríguez, B. A. (2017). Simulation-optimization model for production planning in the blood supply chain. Health care management science, 20(4), 548-564.

Ozdamar, L., \& Yazgac, T. (1999). A hierarchical planning approach for a production-distribution system. International Journal of Production Research, 37, 37-59.

Pant, K., Yadav, V. S., \& Singh, A. R. (2021). Design of multi-tier multi-time horizon closed-loop supply chain network with sustainability under uncertain environment for Indian paper industry. International Journal of Sustainable Engineering, 14(2), 107-122.

Pasandideh, S. H. R., Niaki, S. T. A., \& Asadi, K. (2015). Optimizing a bi-objective multi-product multiperiod three echelon supply chain network with warehouse reliability. Expert Systems with Applications, 42(5), 2615-2623.

Peace, G. S. (1993). Taguchi methods: a hands-on approach: Addison Wesley Publishing Company.

Rafiei, H., Safaei, F., \& Rabbani, M. (2018). Integrated Production-Distribution Planning Problem in a Competition-Based Four-Echelon Supply Chain. Computers \& Industrial Engineering, 119(8599).

Ramezani, M., Kimiagari, A. M., Karimi, B., \& Hejazi, T. H. (2014). Closed-loop supply chain network design under a fuzzy environment. Knowledge-Based Systems, 59, 108-120.

Rezaeian, J., Haghayegh, S., \& Mahdavi, I. (2016). Designing an Integrated Production/Distribution and Inventory Planning Model of Fixed-life Perishable Products. Journal of Optimization in Industrial Engineering, 9(19), 47-60. 
Sarrafha, K., Rahmati, S. H. A., Niaki, S. T. A., \& Zaretalab, A. (2015). A bi-objective integrated procurement, production, and distribution problem of a multi-echelon supply chain network design : A new tuned MOEA. Computers \& Operations Research, 54, 35-51.

Scott, J. R. (1995). Fault tolerant design using single and multi-criteria genetic algorithms. Massachusetts Institute of Technology,

Seyedhosseini, S. M., \& Ghoreyshi, S. M. (2015). An integrated production and distribution planning model for perishable products. International Journal of Operational Research, 23(3), 268-283.

Sokolowski, J. A., \& Banks, C. M. (2010). Modeling and simulation fundamentals: theoretical underpinnings and practical domains (Modelling and Simulation Fundamentals: Theoretical Underpinnings and Practical Domains, Wiley \& Sons Inc., New Jersey.): John Wiley \& Sons.

Srivastava, S. K. (2008). Network design for reverse logistics. Omega, 36(4), 535-548.

Varthanan, P. A., Murugan, N., \& Kumar, G. M. (2012). A simulation based heuristic discrete particle swarm algorithm for generating integrated production-distribution plan. Applied Soft Computing, 12(9), 3034-3050.

Wei, W., Guimarães, L., Amorim, P., \& Almada-Lobo, B. ( 2017). Tactical production and distribution planning with dependency issues on the production process. Omega, 67, 99-114.

Zamarripa, M., Marchetti, P. A., Grossmann, I. E., Singh, T., Lotero, I., Gopalakrishnan, A., et al. (2016). Rolling horizon approach for production-distribution coordination of industrial gases supply chains. Industrial \& Engineering Chemistry Research, 55(9), 2646-2660.

Zheng, Y., Zhang, G., Han, J., \& Lu, J. (2016). Pessimistic bilevel optimization model for risk-averse production-distribution planning. Information Sciences, 372, 677-689.

Zitzler, E., \& Thiele, L. (1999). Multiobjective evolutionary algorithms: a comparative case study and the strength Pareto approach. IEEE transactions on evolutionary computation, 3(4), 257-271. 